\title{
European Hair Research Society 12th Annual Meeting
}

June 29-July 1, 2006, Imperial College, London
Guest Editor

Andrew G. Messenger, Sheffield

\section{Contents}

Editorial

Ebling Lecture

Invited Lecture

Oral Presentations

Poster Presentations

Author Index 80 


\title{
Editorial
}

\author{
Andrew G. Messenger \\ Department of Dermatology, Royal Hallamshire Hospital, Sheffield, UK
}

Welcome to the 12th Annual Meeting of the European Hair Research Society held at Imperial College in London. With delegates from five continents, the meeting promises to be both informative and enjoyable. Over 70 scientific communications are in the programme, including some of the most recent studies on basic hair biology, genetics, model systems, pharmacology, cosmetic science and clinical aspects of hair disease. As well as oral and poster presentations of submitted abstracts, there will be workshops on current topics in the fields of hair follicle neuroendocrinology, hair follicle and epidermal interactions, chemotherapy alopecia and scalp health. The Society is delighted to have Professor Fiona Watt as the John Ebling Lecturer in 2006 with the title 'Generating new hair follicles in adult epidermis'. Professor John Manning will give our other invited lecture on the topic of digit ratios and sex-dependent traits. We warmly thank all those involved in organising the meeting and all the companies who have provided sponsorship of this important forum for scientific interchange.

Andrew G. Messenger

\section{Board of the EHRS}

Andrew Messenger

Rolf Hoffmann

Des Tobin

Pia Freyschmidt-Paul

Kevin McElwee

Ralph Trüeb

\section{Local Organising Committee}

Andrew Messenger

Pattie Birch

Chris Gummer

Andrew McDonagh

Mike Philpott

Valerie Randall

Hugh Rushton

Rachid Tazi-Ahnini

Des Tobin

Gill Westgate

\section{Acknowledgement}

This programme of abstracts was printed with a grant from Unilever 


\section{Ebling Lecture}

\section{Making New Hair Follicles in Adult Epidermis}

F.M. Watt

CR-UK London Research Institute, 44 Lincoln's Inn Fields, London, UK

Adult mammalian epidermis is maintained by stem cells, which self-renew and produce progeny that differentiate along the hair follicle, sebaceous gland and interfollicular epidermal lineages. Two important regulators are Myc and $\beta$-catenin. Myc activation causes stem cell depletion and differentiation into interfollicular epidermis and sebaceous gland. $\beta$-catenin controls lineage choice in a dosedependent fashion and acts, at least in part, by upregulating Hedgehog and Notch signalling. By exploiting Tamoxifen-inducible epidermal transgenes my lab is investigating the consequences of modulating the strength and duration of individual signals, and how different signalling pathways intersect. We are examining the mechanisms by which Myc exerts different effects on stem cells and differentiated cells, and examining whether stem cells can be generated from committed progenitors. The epidermis is an experimentally tractable tissue that provides a paradigm for understanding how stem cell number and lineage selection are controlled in other adult tissues.

\section{Invited Lecture}

\section{2nd to 4th Digit Ratio: A Correlate of Prenatal Testosterone and Sex Dependent Traits}

J. Manning

Department of Psychology, University of Central Lancashire, UK

Sex dependent traits such as male pattern baldness are often influenced by genes and by sex steroids such as testosterone (T). The production of $\mathrm{T}$ begins in the male foetus at around week 8 and peaks at about week 13 . Thereafter it reduces, shows a transitory increase after birth, and is then at low levels until puberty. It is thought that foetal T has major effects on the organisation of many tissues (e.g. the skin) and organ systems (e.g. the brain), and the activational effects of adult $\mathrm{T}$ may be determined by early exposure to prenatal $\mathrm{T}$. Here I introduce a probable proxy for prenatal $\mathrm{T}$, the relative lengths of the 2nd (index) and 4th (ring) fingers or 2D:4D. There is evidence that $2 \mathrm{D}: 4 \mathrm{D}$ is highly conserved in that it is sexually dimorphic in mammals, birds and reptiles. In humans 2D:4D ratio is dimorphic such that males have lower mean 2D:4D than females, the sex difference appears in foetuses as young as 9 weeks, and although there are ethnic differences in 2D:4D the sex dimorphism is universal in humans. Low 2D:4D, particularly low right hand 2D:4D, is related to high in utero $T$ and right - left hand 2D:4D may be positively associated with sensitivity to $\mathrm{T}$ as indicated by the structure of the androgen receptor gene. I discuss how the correlates of 2D:4D range from sex dependent behaviours such as handedness, strength and running speed, to measures of sperm function and family size, and to predispositions to diseases such as toxoplasmosis, eczema, and myocardial infarction.

\section{KARGER}

(C) 2006 S. Karger AG, Basel

Fax +4161306 1234

E-Mail karger@karger.ch

www.karger.com 


\section{Oral Presentations}

\section{1}

\section{Cultured Human Dermal Papilla Cells Induce Hair Formation}

\author{
J. Qiao, E. Philips, J. Teumer \\ Intercytex Ltd., Woburn, MA, USA
}

Follicular Cell Implantation (FCI) is an experimental cell therapy that uses cultured hair follicle cells to induce new hair formation for the treatment of hair loss. This treatment is based on the demonstration that adult dermal papilla cells (DPC) retain the hair inductive capacity they acquired during hair morphogenesis in the embryo. For FCI, hair inductive cells are isolated from scalp biopsies and then propagated in culture in order to provide enough cells to generate many new follicles from a few donor follicles. Following culture expansion, the cells are implanted into the scalp where they induce the formation of new follicles. We have developed a process for FCI that uses a specialised culture medium for cell propagation that preserves the cells' hair inductive capability while allowing significant expansion. In this study, we demonstrate hair induction by human DPC propagated in culture under this process. Using a novel graft assay, we show that DPC are required for hair formation and that the grafted DPC form the dermal papillae of induced hair follicles. The induced hair follicles are histologically normal and are able to grow and cycle for at least 1 year after grafting.

\section{2 \\ Long Term Culture of Mouse Vibrissal Dermal Papilla Cells and Hair Follicle- Inducing Ability \\ A. Osada ${ }^{1}$, T. Iwabuchi ${ }^{2}$, J. Kishimoto ${ }^{2}$, T.S. Hamazaki ${ }^{1}$,
H. Okochi ${ }^{1}$ \\ ${ }^{1}$ Department of Tissue Regeneration, Research Institute, International Medical Center of Japan, ${ }^{2}$ Skin Biology Research Labs, Life Science Research Center, Shiseido Co., Ltd., Japan}

Large numbers of dermal papilla cells are needed for transplantation with epidermal cells to induce de novo hair follicles. However, dermal papilla cells under normal culture conditions do not proliferate well and lose their hair follicle-inducing capacity after more than 10 passages. Therefore, simpler and more stable culture methods for dermal papilla cells have been long desired. When explants of mouse vibrissa dermal papillae were cultured with $10 \%$ FBS-DMEM including bFGF, the outgrowth of dermal papilla cells was markedly stimulated. Moreover, the proliferation of dermal papilla cells was maintained during serial cultivations, and more than 30 passage cultivations were established. To examine their follicle-inducing ability, these established dermal papilla cells were mixed with epidermal cells and injected subcutaneously into nude mice. New hair follicles were induced when dissociated dermal papilla cells at earlier passages (under passage 4) were injected with epidermal cells isolated from newborn or embryonic mice skin, but the cells from higher passages could not induce follicles, as previously reported. Next, we generated spheres by aggregating dermal papilla cells in advance and injected them with epidermal cells. Surprisingly, the spheres made from the higher passage cells (maximum 26 passages) did induce new hair follicles. The expression of several genes specific for dermal papillae in vivo was elevated in the spheres compared with that in adhered dermal papilla cells. These results suggest that bFGF is essential for dermal papilla cell culture and that sphere formation partially represents the intact dermal papilla, resulting in hair follicle induction even by highly passaged cells.

\section{3}

See Congress Programme.

\section{4 \\ Degradation of the Hair Follicle Inner Root Sheath \\ G. Westgate ${ }^{2}$, M. Blount ${ }^{1}$, P. Slusareewicz ${ }^{1}$, P. Parmar', S. Goff', \\ 'Unilever R\&D Colworth, Sharnbrook, Bedford, ${ }^{2}$ Westgate Consultancy, Stevington, Bedfordshire, UK}

A widely held belief in hair research is that degradation of the inner root sheath (IRS) is mediated by a function of the sebaceous gland. This belief has been supported by the fact that isolated human hair follicles lacking sebaceous glands grow fibres in culture to which the root sheaths appear to remain attached. Here we report that this is not the case. The fibres of follicles lacking sebaceous glands, when grown in culture, are encased in a layer of translucent tissue. During hair growth in vitro this tissue remains intact at the distal end of the follicle but appears to be absent further down towards the bulb. Transaction within the region lacking this tissue results in the release of a naked hair fibre and the production of hair with no attached sheath tissue upon further growth. Additionally by utilising a serial sectioning technique we demonstrate that the translucent tissue does in fact represent the IRS and that this tissue is indeed degraded in vitro in the absence of the sebaceous gland. Finally by histological comparison with freshly isolated pilosebaceous units indicates that IRS degradation in vitro strongly resembles the process that occurs in vivo. Taken together these data suggest that current thought regarding the mechanism of IRS degradation is flawed and indicate that the sebaceous gland does not itself participate in this process. IRS degradation appears to be a function of the follicle itself and is probably 
intimately linked with the processes of cellular differentiation that occur during hair biogenesis. These data further validate the human hair follicle culture model with respect to its ability to reproduce the processes of hair formation, which occur in vivo.

\section{O5 \\ BMP Signaling Regulates Size of Hair Follicles via Modulating the Expression of Cell Cycle-Associated Genes}

\author{
A. Sharov, T. Sharova, R. Atoyan, A. Mardariev, V. Botchkarev
}

Department of Dermatology, Boston University School of

Medicine, USA

Bone morphogenetic protein (BMP) signaling is involved in regulation of a large variety of developmental programs including those controlling organ size. Here, we show that transgenic mice overexpressing BMP antagonist noggin (promoter: K5) are characterised by marked increase of size of the anagen hair follicles (HFs) and by replacement of zig-zag hairs by awl-like hairs, compared to the agematched wild-type controls. In K5-Noggin mice, increase in size of the hair bulb is not accompanied by any significant changes in size or cellularity of the follicular papilla. However, markedly enlarged anagen HFs of K5-Noggin mice show increased proliferation in the matrix and increased number of the hair cortex and medulla cells, compared to wild-type HFs. Microarray and real-time PCR analyses of the laser captured hair matrix cells show strong decrease in expression of cyclin-dependent kinase inhibitor p27Kip1 and increased expression of selected cyclins in K5-Noggin vs. wild-type mice. Similarly to K5-Noggin mice, p27Kip1 knockout mice also show increased size of anagen HFs associated with increased cell proliferation in the hair bulb. Activation of BMP signaling in HaCaT keratinocytes induces growth arrest, stimulates p27Kip1 expression and positively regulates $\mathrm{p} 27 \mathrm{Kip} 1$ promoter activity, thus further supporting a role of $\mathrm{p} 27 \mathrm{Kip} 1$ in mediating the effects of BMP signaling on HF size. These data suggest that BMP signaling plays important role in regulating cell proliferation and controls size of anagen HFs via follicular papilla-independent modulation of the expression of cellcycle associated genes in hair matrix keratinocytes.

\section{6}

\section{Involvement of Edar Signaling in the Control of Hair Follicle Involution (Catagen)}

\section{Fessing, T. Sharova, A. Sharov, R. Atoyan, V. Botchkarev \\ Department of Dermatology, Boston University School of Medicine, USA}

Ectodysplasin (Eda) and its receptor (Edar) are required for normal development of several ectodermal derivatives including hair follicles (HFs). Here, we show that during murine hair cycle the expression of EdaA1, Edar, EDARADD and TRAF6 transcripts are minimal in the resting phase and maximal during HF transition from active growth to the regression (catagen). EdaA1 mRNA and Edar proteins are expressed in the hair matrix, outer and inner root sheaths of anagen HFs. During catagen, EdaA1 mRNA and Edar protein are expressed in the outer and inner root sheaths and later in the secondary hair germ. Catagen development accompanied by significantly increased number of TUNEL-positive cells in the outer root sheath is significantly accelerated in downless mice or after treatment of wild-type mice by fusion protein that inhibits Edar signaling, compared to the corresponding controls. Microarray, real-time PCR and immunohistochemical analyses of skin of downless mice reveal strong decrease of expression of X-linked inhibitor of apoptosis protein (XIAP), compared to the controls, suggesting XIAP as a target for Edar signaling. Thus, our data demonstrate that in addition to its well-established role in HF morphogenesis, Edar signaling is also involved in hair cycle control at least in part by regulating apoptosis in HF keratinocytes during catagen.

07

\section{The Expression of Early Markers of Adipocyte Differentiation in Sebaceous Gland Cells \\ W. Harrison', J.J. Bull', C.C. Zouboulis', M.P. Philpott \\ ${ }^{1}$ Centre for Cutaneous Research, Barts and The London \\ Queen Mary's School of Medicine and Dentistry, University \\ of London, London, UK; ${ }^{2}$ Department of Dermatology, \\ University Medical Centre Benjamin Franklin, The Free \\ University of Berlin, Berlin, Germany}

The transcription factors CCAAT enhancer binding protein (C/EBP) $\alpha, \beta$ and $\Delta$, and peroxisome proliferator-activated receptor (PPAR) $\gamma$ are known to have a crucial role in the differentiation of adipocytes and are expressed in sebaceous gland cells [1,2]. This has led us to believe that sebocytes may follow a similar program of differentiation to adipocytes, with lipid synthesis and accumulation common to both cell types. As our understanding of adipocyte differentiation is more advanced than that of sebocytes, common pathways between the two cell types could be of significant advantage to understanding of sebocyte biology. Using RT-PCR, immunohistochemisty and western blotting we have looked for the expression of known early markers of adipocyte differentiation including resistin, galectin-12, SREBP-1 and stearoyl-CoA desaturase in the immortalised sebocyte cell line SZ95, keratinocytes and whole skin. The expression of resistin, galectin-12, SREBP-1 and stearoyl-CoA desaturase has been detected as both mRNA and protein in cultured sebocyte cell line SZ95, keratinocytes and whole skin. Distinct immunoreactivity for resistin, galectin-12 and SCD is found throughout the cytoplasm of both sebocytes and to a lesser extent keratinocytes. Whilst for SREBP-1 the immunoreactivity is predominantly in the nucleus of sebocytes. This study shows that exvivo and in-vitro cultured sebocytes express factors associated with early differentiation of adipocytes, adding to the evidence that sebaceous gland cells undergo a process of differentiation similar to adipocytes. We therefore, propose that further characterisation of sebocyte differentiation based on published adipocyte research may rapidly advance our understanding of sebocyte biology.

\section{References}

1 Chen WJ: Invest Dermatol 2003;121:441-447.

2 Bull JJ: J Invest Dermatol 2002;118:17-24 
08

\section{Dihydrotestosterone (DHT)-Inducible DICKKOPF 1 from Scalp Dermal Papilla Cells Causes Apoptosis in Follicular Keratinocytes}

Y.-K. Sung, M.-H. Kwack, M.-K. Kim, J.-C. Kim

Department of Immunology, School of Medicine, Kyungpook

National University, Daegu, Korea

Paracrine factors induced by dihydrotestosterone (DHT) are thought to influence the activity of the follicular cells. RT-PCR analysis and ELISA showed that DICKKOPF 1 (DKK-1) is up-regulated and secreted to conditioned media upon DHT exposure. In addition, in an vitro co-culture system by using dermal papilla cells (DPCs) and outer root sheath keratinocytes (ORS) we showed that DHT inhibits ORS growth and neutralizing antibody against DKK-1 significantly attenuates DHT-induced growth inhibition of ORS cells. Flow cytometry analysis showed DHT induces substantial apoptosis in co-cultured ORS and caspase inhibitor attenuates DHT-induced growth inhibition of ORS. Altogether, this study shows that secreted DKK-1 from DPCs by DHT causes apoptosis in keratinocytes and suggests that DHTinducible DKK-1 is responsible for the hair growth inhibition.

\section{9}

\section{Human Hair Follicle and Dermal Fibroblasts have Different Responses to Oestrogens after Wounding in Vitro}

\author{
S. Stevenson', L. Nelson' ${ }^{2}$ S. Huq ${ }^{1}$, S. Adekunle', \\ K. Asaad', D. Sharpe1, M.J. Thornton ${ }^{2}$ \\ ${ }^{1}$ Burns and Plastic Surgery Research Unit, ${ }^{2}$ Department of \\ Biomedical Sciences, University of Bradford, Bradford, UK
}

Improved wound healing in hairy skin is thought to involve hair follicle fibroblasts. Wound healing is delayed in post-menopausal women, but is improved with estrogen therapy. Since we have previously reported that human dermal fibroblasts and dermal papilla cells express estrogen receptors, we have evaluated the migration, proliferation and collagen secretion of cultured breast and scalp dermal fibroblasts, and scalp follicular dermal papilla and dermal sheath cells in response to 17- $\beta$-oestradiol following mechanical wounding. Primary cultures of dermal fibroblasts $(n=8)$, dermal sheath $(n=4)$ and dermal papilla cells $(n=4)$ were established from female scalp skin or from breast skin (fibroblasts only $n=5$ ). Cells were mechanically wounded and incubated in serum-free, phenol red-free medium $\pm 10 \mathrm{nM} 17-\beta$-oestradiol. Migration was evaluated using a scratch-wound assay, DNA synthesis by $3 \mathrm{H}$-thymidine uptake and collagen secretion by the Sircol assay. Post wounding, all cells demonstrated a significant increase in DNA synthesis. Dermal fibroblasts derived from breast showed a further significant increase in response to $17-\beta$-estradiol. Post-wounding, scalp fibroblasts and dermal sheath cells migrated at similar rates, significantly faster than breast fibroblasts. However, the rate of migration of the dermal papilla cells was significantly faster than all groups. Incubation with $17-\beta$-oestradiol significantly increased the migration of both breast and scalp dermal fibroblasts, but had no effect on hair follicle fibroblasts. Wounding significantly increased the secretion of collagen by breast and scalp fibroblasts; $17-\beta$-oestradiol had no effect on collagen secretion by any of the cell lines. These results demonstrate that wounding stimulates migration and proliferation in cultured follicular fibroblasts, supporting the hypothesis that these cells play a role in wound healing. Furthermore, following wounding estrogen directly stimulates dermal fibroblast proliferation and migration in vitro, supporting the improved wound healing rates seen with estrogen therapy. Whether 17- $\beta$-oestradiol has indirect effects on hair follicle cells is currently under investigation.

\section{0}

\section{Evaluation of Androgens and Cholesterol in the Scalp Hair and Plasma of Patients with Male-Pattern Baldness Before- and After- Finasteride}

W.-Y. Sim', B.-L. Lew', H.K. Ryu' ${ }^{2}$, K.M. Kim², B.C. Chung ${ }^{2}$

${ }^{1}$ Department of Dermatology, College of Medicine, Kyung Hee University, ${ }^{2}$ Bioanalysis and Biotransformation Research Center, KIST, Seoul, Korea

Finasteride, a competitive inhibitor of 5 - $\alpha$-reductase II enzyme, is widely used as a medical treatment for patients with male-pattern baldness (MPB), which is affected by the distribution of androgenic steroids. To study the effect of finasteride, androgenic steroids and cholesterol in the vertex and occipital scalp hair and in the plasma of patients with MPB were quantified. The patients with MPB, aged 23-52 years, were treated with $1 \mathrm{mg}$ daily of finasteride for 5 months. The hair and plasma samples were hydrolyzed, extracted with n-pentane, and derivatised with MSTFA:NH4I:DTE (1000:4:5, v/w/w). We analyzed the concentrations of dihydrotestosterone (DHT) and testosterone $(\mathrm{T})$ in the hair and plasma using gas chromatography-mass spectrometry (GC-MS). In the case of the hair, the ratio of DHT/T was decreased in the vertex scalp hair after the individual received the finasteride dosage $(p<0.005)$. However, we found no significant difference in the ratio of DHT/T in the occipital scalp hair before and after the individuals received the finasteride dosage. Like the results in the vertex scalp hair, the ratio of DHT/T in the plasma was remarkably decreased after finasteride dosage $(p<0.001)$. Interestingly, cholesterol was increased after finasteride in the hair of vertex and occipital scalp $(p<0.001)$. This study supports the effect of finasteride in patients with MPB by the examination of decreased level of $\mathrm{DHT} / \mathrm{T}$ on scalp hair and in plasma.

\section{1}

Microscopic Hair Characteristics: A Tool for Dealing with Wildlife Offence Cases in India

\section{Sahajpal, S.P. Goyal}

Wildlife Forensic Cell, Wildlife Institute of India, Dehradun, Uttranchal, India

The value of hair as physical evidence is well appreciated and acknowledged in dealing with crime cases. Microscopic studies on hair have been used in identification of species and individuals. 
Microscopic structure of hair (cuticle, medulla, cross-section etc.) is of immense value in identifying mammalian species in wildlife offence cases. India is home to over 400 species of mammals. Most of these mammals are facing extinction due to illegal poaching and illegal trade in their parts and products. The major reason behind this flourishing illegal trade is lack of know-how and reference data to identify species from physical evidence (mostly hair) in case of wildlife offence cases related to mammals, as such the poachers escape conviction under wildlife protection laws. Since the mammalian species are most prone to poaching and hair is the most common physical evidence found in such cases, we characterised hair of 57 Indian mammal species, most prone to poaching using microscopic techniques (Leica DMR for light and Philips for SEM). The colour, texture, length, thickness, medulla thickness, medulla type, medulla percentage, cross-section shape, cuticular pattern (proximal, medial and distal) were studied. The observations were statistically evaluated with statistical software like SPSS-8 and Statistica. All the species could be successfully characterised with microscopic studies by using various parameters like medulla, cuticle and cross section etc. All the observations for these 57 species will be compiled in the form of a reference guide, which can be used for species identification by crime investigation agencies, forensic labs and people working on trichotaxonomy. In addition to this we report keratin profiles of some highly endangered mammalian species using SDS-PAGE (sodium dodecyl sulphate polyacrylamide gel electrophoresis) and IEF (Iso electric focusing).

\section{2 \\ Oral Ciclosporine in Lichen Planopilaris \\ P. Reygagne, P. Assouly, B. Matard, C. Lyonnet, \\ C. Jouanique, I.R. Hasnaoui \\ Centre Sabouraud, Hopital Saint-Louis, Paris, France}

Lichen planopilaris (LPP) is a rare inflammatory disease that causes permanent alopecia. When the disease is very active the best treatment is oral corticotherapy, but relapse is frequent. A short course of oral cyclosporine has been reported successful in treating 3 patients. We present 12 patients with refractory LPP treated for 3-7 months with cyclosporine. They were aged from 31 to 59 years with a diagnosis of LPP from 1 to 19 years. Previous treatment included topical and intralesional corticosteroids, hydroxychloroquine, chloroquine, topical tacrolimus and oral corticotherapy $(n=5)$. At the beginning of the study, the dosage of cyclosporine was $3 \mathrm{mg} / \mathrm{kg} /$ day, increased monthly in case of non-response. Treatment was stopped after 2 months without symptoms. In a few patients we measured hair density on a selected target and performed global standardised photography. We obtained complete clinical resolution of the disease activity at doses ranging from 250 to $400 \mathrm{mg}$, and treatment duration of 4-8 months. A complete clinical response was achieved in 8 patients and a partial response in 2 . Two patients failed to respond. Six months after stopping cyclosporine 6 patients remained symptom free and 4 had relapsed. Hair count was available before and after treatment for 9 patients with stabilisation $(n=4)$, increase $(n=3)$ or decrease $(n=2)$. The two patients with a decrease were clinically rated failure or partial response. Side-effects were minor: these included transient elevation of serum creatinine $(n=4)$, distal paraesthesia (4), and hypertrichosis (2). On the basis of this experience:

- Cyclosporine can be an option to treat refractory LPP;

- Optimal efficient dosage is between 4 and $5 \mathrm{~m} / \mathrm{kg} /$ day;

- Optimal course is 4 months;

- Success rate is $83 \%$;

- Relapse rate 6 months after stopping the course is $40 \%$;

- A study vs. systemic oral corticotherapy is necessary.

013

\section{Follicular Targeting - Implementation in Transcutaneous Vaccination}
A. Vogt ${ }^{1}$, B. Combadiere', B. Mahe ${ }^{2}$, S. Hadam ${ }^{1}$, K.M. Stieler ${ }^{1}$ J. Lademann', H. Schaefer', B. Autran², W. Sterry',
U. Blume-Peytavi ${ }^{1}$
${ }^{1}$ Clinical Research Center for Hair and Skin Physiology, Department of Dermatology and Allergy, Charite - Universitätsmedizin Berlin, Germany; ' Laboratoire d' Immunologie Cellulaire et Tissulaire, Hopital Pitie Salpetriere, Universite Pierre et Marie Curie, Paris, France

Due to the unique role of the hair follicle in percutaneous penetration, drug delivery systems which target active compounds to the hair follicle, may result in a better penetration and a higher efficiency of hair and skin therapy ('Follicular Targeting'). Applications in immunotherapy, e.g. transcutaneous vaccination, are of particular interest, because skin antigen-presenting cells (APCs) can be found at particularly high densities in hair follicle-bearing skin, where they are concentrated around the upper portion of the hair follicles. Our in vitro studies on human skin revealed, that nanoparticles, due to their ability to aggregate in the hair follicle openings and to penetrate along the follicular duct, are promising carrier systems for transfollicular drug delivery. Transcutaneously applied nanoparticles in the size range of $40 \mathrm{~nm}$, moreover, were capable of penetrating the epithelium and entered epidermal LCs, suggesting that such particles may be used to transcutaneously deliver active vaccine compounds, via the hair follicle, into cutaneous APCs. To further assess the potential of particle-based vaccines in transcutaneous vaccination, we developed a standard operating procedure (SOP) for transcutaneous vaccine delivery based on our current knowledge on follicular penetration. In a limited number of volunteers, we found that this newly developed $\mathrm{SOP}$ is safe and efficient at inducing immune responses.

\section{4 \\ The Use of Placebo or Untreated Controls in Male Pattern Hair Loss Studies Leads to a Significant and Permanent Loss of Hair \\ D.H. Rushton 1, I.D. Ramsay², J.J.H. Gilkes ${ }^{2}$ \\ ${ }^{1}$ School of Pharmcay \& Biomedical Sciences, University of \\ Portsmouth, Portsmouth, ${ }^{2}$ Lister Hospital, London, UK}

Do men with male pattern hair loss (MPHL) irreversibly lose hair if they are assigned to a placebo or untreated group? Monozygotic 
twins with MPHL, who were clinically indistinguishable for extent of hair loss and medical history, were followed for 4 years. During the first year, twin $1(\mathrm{OW})$ was treated with an effective topical type I $5-\alpha$ reductase inhibitor in combination with minoxidil and oral finasteride; a type II $5-\alpha$ reductase inhibitor. Twin $2(\mathrm{RW})$ remained untreated for 12 months following which he received the same treatment as his brother. After 36 months on treatment a unit area trichogram was undertaken in the same frontal area in each twin. Comparing both twins after 36 months treatment, a significant decrease $(\mathrm{p}<0.05)$ in Total Hair Density (THD: $247 \mathrm{hair} / \mathrm{cm}^{2}$ vs. $212)$ and $(\mathrm{p}<0.01)$ in Useful Hair Density (UHD: 140 hair $>30 \mathrm{~mm}$ in length $/ \mathrm{cm}^{2}$ vs. 104) was found in twin 2(RW). A significant $(\mathrm{p}<0.05$, one tail) decrease in mean hair diameter $(79 \mu \mathrm{m}[\mathrm{OW}]$ vs. $73 \mu \mathrm{m}$ [RW]) of hair longer than $30 \mathrm{~mm}$ in length and an increase in hair unable to grow beyond $30 \mathrm{~mm}$ was also found in twin 2(RW), who started treatment 1 year later than his brother(OW). On initial presentation these identical twins were clinically indistinguishable and we believe had no difference in baseline hair status. A chance event resulted in twin 2(RW) remaining untreated for 12 months. In our experience we would not expect to see any further improvement after 3 years treatment. Consequently, these data indicate that hair loss progressed in the untreated twin 2(RW) and that these changes were irreversible despite receiving a known efficacious regimen. Delaying treatment, such as employing placebo or untreated controls in clinical trials where an active control is available, appears unethical and needs reviewing.

\section{5 \\ Detection of Functionally Active Melanocortin Receptors and Evidence for an Immunoregulatory Activity of Alpha-Melanocyte-Stimulating Hormone in Human Dermal Papilla Cells}

\section{Boehm}

Department of Dermatology, University of Munster, Munster, Germany

Proopiomelanocortin (POMC)-derived peptides and their receptors have been identified in many peripheral organs including the skin in which they exert a diversity of biological actions. We investigated the expression and potential role of the POMC system in human dermal papilla cells (DPCs), a specialised cutaneous mesenchymal cell type regulating hair follicle activity. In culture, these cells expressed POMC and displayed immunoreactivity for $\mathrm{ACTH}, \alpha-\mathrm{MSH}$, and $\beta$-endorphin. Among the prohormone convertases (PCs) tested, only PC2, its chaperone $7 \mathrm{~B} 2$, and furin convertase but not $\mathrm{PC} 1$ and paired basic amino acid cleaving enzyme 4 gene were detected. Human DPCs in vitro expressed both the melanocortin-1 receptor (MC-1R) and $\mathrm{MC}-4 \mathrm{R}$, and immunoreactivity for these receptors was also present in cells of the human dermal papilla in situ. In contrast to the dermal papilla of agouti mice, agouti signaling protein, a natural and highly selective MC-1R and MC-4R antagonist, was undetectable in human DPCs. The MC-Rs detected in human DPCs were functionally active because $\alpha$-MSH increased intracellular cAMP and calcium. Preincubation of the cells with a synthetic peptide corresponding to the $\mathrm{C}$-terminal domain of agouti signaling protein abrogated cAMP induction by $\alpha$-MSH. Furthermore, $\alpha$-MSH was capable of antagonizing the expression of intercellular adhesion molecule-1 induced by the proinflammatory cytokine interferon- $\gamma$. Our data suggest a regulatory function of $\alpha$ MSH within the dermal papilla whose disruption may lead to deregulation of immune and inflammatory responses of the hair follicle, thereby possibly contributing to the development of inflammatory forms of alopecia.

\section{6 \\ Functional Evidence Supporting the Use of Human Scalp Melanocytes in Neurodegeneration Research: Cytotoxicity of Amyloid Beta Peptides and Interactions between Amyloid Precursor Protein and the Adaptor Protein Fe65}

\author{
N. Papageorgiou, E. Carpenter, D.J. Tobin \\ Medical Biosciences, School of Life Sciences, University of \\ Bradford, Bradford, UK
}

The neurodegenerative disorder Alzheimer's disease (AD) is an increasing financial and social burden to ageing populations of the developed world. AD pathology is associated with the extracellular deposition of amyloid $\beta$ peptides ( $A \beta 40$ and $A \beta 42$ ), leading to the formation of senile plaques in affected brains. These peptides are cleaved from the amyloid precursor protein (APP) by $\beta$ - and $\gamma$-secretases. It has been suggested that cutaneous melanocytes may be a useful proxy for neurones in neurodegeneration research due to their sharing a close embryonic origin and multiple other similarities. In this study we examined the expression of neuron-associated proteins in cultured follicular and epidermal melanocytes and tested these cells in vitro under conditions similar to those found in AD. Human scalp melanocytes were incubated with increasing concentrations $(1 \mu \mathrm{M}, 10 \mu \mathrm{M}$ and $30 \mu \mathrm{M}$ ) of aggregated (confirmed by native 16\%-PAGE) A $\beta 40$ peptide. Results showed a significant reduction in melanocyte viability

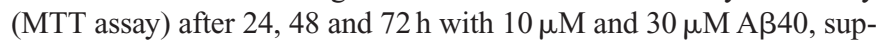
porting a functional similarity between melanocytes and neurones in the context of $\mathrm{AD}$. As the intracellular domain fragment of amyloid $\beta$ precursor protein is thought to activate Fe65-dependent gene expression in $\mathrm{AD}$, we also examined whether this may also occur in melanocytic cells by probing melanoma nuclei for the adaptor protein Fe65 and the last 15 amino acids of the C-terminus of APP (APPCT15). These studies revealed the presence of both proteins in melanoma nuclei, suggesting their potential for interaction with subsequent Fe65dependent gene transactivation, potentially also possible in cutaneous cells. We are currently examining whether Fe65 and APPCT15 exist as a complex in melanocytes, including hair follicle melanocytes (i.e. can be co-immunoprecipitated), as this would further support the potential use of cutaneous melanocytes as a neuronal proxy in $\mathrm{AD}$ and neurodegeneration research. 
017

\section{Proopiomelanocortin (POMC)-Derived Peptides and Corticotropin-Releasing Hormone (CRH) Influence the Behaviour of Human Scalp Follicular and Dermal Fibroblasts by Modulating Cell Proliferation and Transforming Growth Factor-Beta (TGF-Beta)-Associated Collagen}

\author{
S. Huq, D. Sharpe, R. Karoo, S. Stevenson, S. Adekunle, \\ K. Asaad, D.J. Tobin \\ Plastic Surgery and Burns Research Unit, School of Life \\ Sciences, University of Bradford, UK
}

The skin both produces and is targeted by POMC and CRH peptides. Recently Bohm et al. reported that $\alpha$-melanocyte stimulating hormone $(\alpha-\mathrm{MSH})$ can antagonise TGF- $\beta$-induced collagen production in dermal fibroblasts. Moreover, Jahoda et al. have suggested that follicular fibroblasts may be a more effective wound healing cell. The purpose of the study was to characterise the expression of POMC and $\mathrm{CRH}$ peptides in fibroblast subpopulations and to investigate their effects on cell proliferation and collagen secretion. Fully matched sets of inter-follicular (DF), and follicular fibroblasts (dermal sheath-DS and follicular dermal papilla-DP) were obtained from the scalp of 5 normal healthy females (49-60years, passage 4-6) and cultured using conventional methodologies. The expression of POMC and $\mathrm{CRH}$ peptides, associated receptors (MC1-R, MOR and CRH-R1) and pro-hormone convertases (PC1 and PC2/7B2) were investigated using specific antibodies on serum-starved cells. Staining incidence and intensity were assessed by image analysis software (Paint ShopPro). Cells were incubated with POMC and CRH peptides $(10-8 \mathrm{M})$ for 5 days followed by assessment of proliferation (MTT assay) and TGF- $\beta$-induced collagen secretion (Sircol assay). These studies were compared with fibroblast subpopulations in a 'wounded'-state (Scratch assay). Inter-follicular and follicular fibroblast sub-populations variably expressed all POMC and CRH peptides, as well as PC1, PC2/7B2 and POMC and CRH peptide receptors. $\alpha-$ $\mathrm{MSH}, \mathrm{ACTH}, \mathrm{CRH}$ (but not $\beta$-endorphin) stimulated the proliferation of all fibroblast subpopulations, compared to unstimulated controls. TGF- $\beta$ stimulated collagen secretion by all fibroblast types and this effect was antagonised by $\alpha-\mathrm{MSH}, \mathrm{ACTH}$ and $\mathrm{CRH}$. Wounding of the fibroblast monolayers revealed increased basal collagen secretion in DS and less so in DF, but not in DP fibroblasts. This study demonstrated that all human scalp fibroblast sub-populations expressed the full POMC and CRH machinery in vitro. Furthermore, these peptides can antagonise TGF- $\beta$-induced collagen secretion suggesting a possible role in cutaneous wound healing.
018

\section{Genetic Analysis of Autoimmune Regulator} (AIRE) Haplotypes in Alopecia Areata

\author{
D. Wengraf ${ }^{1}$, T. Lovewell ${ }^{1}$, M. Cork ${ }^{1,2}$, A.W. Messenger ${ }^{2}$, \\ A. McDonagh ${ }^{2}$, R. Tazi-Ahnini ${ }^{1,2}$ \\ ${ }^{1}$ Division of Genomic Medicine, ${ }^{2}$ Dermatology \\ Immunogenetics Group, University of Sheffield, \\ Sheffield, UK
}

Alopecia areata (AA) is a disorder primarily affecting the hair and nails associated with autoimmune diseases. Autoimmune Polyglandular Syndrome-1 (APS-1) is caused by loss-of-function mutations in the AIRE gene on chromosome 21. There are several associated autoimmune diseases including hypoparathyroidism, thyroid disorders, vitiligo, and diabetes mellitus. In particular, AA has $\sim 40$ times increased prevalence amongst APS-1 patients, suggesting that AIRE is a strong candidate gene for AA. We identified six different SNP's in the AIRE gene to be used in genetic analysis. These were at positions C-103T, C4144G, T5238C, G6528A, T7215C and T11787C. SNP's were genotyped in 295 AA patients and 363 healthy controls using RFLP analysis. The PMPLUS program was used to analyse the different haplotypes of these six polymorphisms. The haplotype analysis was repeated using tagged SNPs (C-103T, G6528A, T7215C and T11787C). The online program, Mfold (Version 3.2) was used to predict secondary mRNA structure for these four SNP haplotypes. All SNPs were in Hardy-Weinberg equilibrium in the control group. Genetic analysis of individual SNPs revealed that the only significant association with $\mathrm{AA}$ was with the $7215 \mathrm{C}$ allele $\left(\mathrm{p}=2 \times 10^{-10}\right)$. Standardised coefficients of disequilibrium were calculated between all pairs of these SNPs and showed that T7215C is in complete linkage disequilibrium (LD) with T-103C and T11787C. Haplotype analysis has shown a highly significant association between AA and the haplotypes CCTGCT and CGTGCC $\left(\mathrm{p}=6.05 \times 10^{-6}\right.$ and $\mathrm{p}=0.001$ respectively), whereas CCTGTC was shown to have a protective effect $(\mathrm{p}=0.004)$. The haplotype anaylsis using tagged SNPs showed two haplotypes having significant associations with AA; these were CGCT and CGCC $\left(\mathrm{p}=3.84 \times 10^{-7}\right.$ and $\mathrm{p}=3.94 \times 10^{-4}$, respectively). The hapolotype CGTC was also shown to have a protective effect $\left(\mathrm{p}=2.10 \times 10^{-4}\right)$. The predicted mRNA structures for the CGTC, CGCC and CGCT haplotypes are different. The haplotype CGCT has the lowest free energy $(\Delta \mathrm{G}-975.75 \mathrm{kcal} / \mathrm{mol})$ suggesting a more stable mRNA structure than for the mRNA of haplotypes CGCC and CGTC ( $\Delta$ G-964.42 kcal $/ \mathrm{mol}$ and $\Delta \mathrm{G}-964.77 \mathrm{kcal} / \mathrm{mol} \mathrm{respec}-$ tively). These findings suggest that the AIRE susceptibility haplotypes may affect the stability of AIRE mRNA which could play a central role in the pathogenesis of AA. 
019

The Impact of CD44 Blockade on Leukocyte Migration in a Skin-Associated Autoimmune Disease Treated by a Contact Allergen

P. Freyschmidt-Paul ${ }^{1}$, Z. Margot ${ }^{2}$, P. Gupta ${ }^{2}$, M. Vitacolonna ${ }^{2}$, S. Hummel²

${ }^{1}$ Department of Dermatology, Philipp University, Marburg,

${ }^{2}$ German Cancer Research Center, Heidelberg, Germany

CD44 plays an important role in leukocyte migration. Anti-CD44 has been shown to mitigate delayed type hypersensitivity (DTH) reactions. In addition, induction of alopecia areata (AA) can be prevented by a CD44v10-specific antibody. On the other hand, AA can be cured by a mild chronic eczema. Taking the therapeutic activity of antiCD44 in AA as well as in DTH reactions, it became of interest to see whether both phenomena are based on the same or different mechanisms, which could give a hint, whether a combination of anti-CD44 treatment and induction of a mild chronic eczema would be contraindicated or could be considered a therapeutic improvement. To answer the question, the impact of anti-panCD44 and anti-CD44v10 was evaluated in repeatedly allergen (SADBE)-treated control and AA-affected mice. Anti-CD44 treatment had no impact on leukocyte activation in allergen-treated control and AA mice. Instead, antipanCD44 and with equal efficacy anti-CD44v10 inhibited leukocyte migration in vivo and in vitro in untreated, AA and allergen treated control and AA mice. Anti-CD44-mediated inhibition of leukocyte migration was not accompanied by down-regulated expression of CD44 or of additional adhesion molecules, required for endothelial cell adhesion and extravasation. Instead, a blockade of CD44v10 interfered with leukocyte, most prominently with monocyte homing into the skin. Exclusively in AA affected mice, a panCD44-specific antibody also prevented $T$ cell entry into the draining lymph node. In allergen-treated AA-affected mice there has been evidence that CD44v10 may additionally interfere with emigration of activated, chemokine receptor expressing $\mathrm{T}$ cells from the draining lymph node. We interpret the data in the sense that anti-CD44 prohibits extravasation of activated leukocytes despite the presence of adhesion molecules, chemokine receptors and chemoattractants. A blockade of CD44v10 selectively interferes with DTH and AA effector cells homing into the skin. A panCD44-specific antibody, though with lower efficacy, can also block leukocyte entry into the lymph node and/or egress of activated $\mathrm{T}$ cells from the draining node, which has mostly been seen in AA-affected mice. Thus, anti-CD44 treatment together with induction of a mild chronic eczema may well improve therapeutic efficacy in AA.
020

Two Stage Model for Induction of Autoimmunity: SEB Synergises with Interferon-Gamma to Induce Alopecia Areata

\author{
A. Gilhar', G. Kaufman'1 , B. Aassi', R.S. Kalish, \\ Department of Dermatology ${ }^{2}$ \\ ${ }^{1}$ Skin Research Laboratory, The B. Rappaport Faculty of \\ Medicine, Technion-Israel Institute of Technology, \\ Flieman Medical Center, Haifa, Israel; '2SUNY at Stony Brook, \\ New York, USA
}

It was hypothesised that autoimmune T-cell diseases require two conditions, loss of tolerance to induce autoreactive T-cells, and a stimulus, such as superantigen, to expand the autoreactive T-cells. Alopecia areata (AA) is associated with loss of immune privilege in hair follicles. It was proposed that treatment with Staphylococcal Enterotoxin $\mathrm{B}$ (SEB) superantigen would expand autoreactive T-cells and synergise with IFN- $\gamma$ to induce $\mathrm{AA}$ in $\mathrm{C} 3 \mathrm{H} / \mathrm{HeJ}$ mice. $\mathrm{C} 3 \mathrm{H} / \mathrm{HeJ}$ mice 7 weeks old were injected IV with murine IFN- $\gamma 2 \times 104 \mathrm{U}$ or PBS for three consecutive days, followed by injections every 7 days. 30 days following the first injection, the female and male mice were divided into three groups: Group 1: (PBS alone) mice injected with PBS for 30 days received additional PBS injections IV for 2 consecutive days followed by injections with PBS every 7 days. Group 2: (IFN- $\gamma$ alone) mice treated 30 days with IFN- $\gamma$ were injected IV with IFN- $\gamma$ for 2 days and followed by every 7 days. Group 3: (IFN- $\gamma$ and SEB). Treated 30 days with IFN- $\gamma$, injected intraperitoneal with SEB $(50 \mathrm{mg})$ for 2 days followed by injections with IFN- $\gamma$ every 7 days. Mice were examined for hair loss. Skin was harvested 14 weeks after the first injection and analyzed by histology as well as immunohistochemistry for ICAM-1, and MHC class I and II. Clinical rates of AA development on back and abdomen of $\mathrm{C} 3 \mathrm{H} / \mathrm{HeJ}$ mice; Group 1: PBS alone 7\% (1/14), Group 2: IFN- $\gamma$ alone 23.5\% (4/17), Group 3: IFN- $\gamma+$ SEB $60 \%(9 / 15),(\mathrm{p}<0.05$ for Group 3 vs. Group 1$)$. IFN- $\gamma+$ SEB was superior to IFN- $\gamma$ alone in inducing AA in a susceptible mouse strain. This supports the hypothesis of a two-stage model for development of autoimmune disease. 


\section{Poster Presentations}

P1

\section{Effect of Thioredoxin Reductase 1 on Glucocorticoid Receptor Activity in Human Outer Root Sheath Cells}

\author{
C.D. Kim, K.H. Kim, S. Jang, D.-K. Choi, M. Im, Y.-J. Seo, \\ J.-H. Lee, J.-K. Park \\ Department of Dermatology, Chungnam National University, \\ Daejeon, Korea
}

Alopecia areata (AA) is a common disease of patchy hair loss on the scalp that can progress to cover the entire scalp and eventually the entire body. Intralesional injection of corticosteroids is the first-line therapy for adult patients, and it is believed that the effect of glucocorticoid is mediated through an immunosuppressive mechanism primarily. Although it is a very powerful treatment for AA, some patients do not respond to glucocorticoid treatment effectively. To delineate the molecular mechanism underlying glucocorticoid insensitivity, we examined the expression of glucocorticoid receptor (GR) and thioredoxin reductase 1 (TrxR1) in AA patients. In some cases of glucocorticoid resistant AA, the expression of TrxR1 was significantly decreased in outer root sheath (ORS). We then investigated the effect of TrxR1 on GR activity using recombinant adenoviruses. The ORS cells were transduced with TrxR1- and GR-expressing adenoviruses together with reporter virus. The results showed that TrxR1 significantly increased GR activity. Next, we determined the effect of TrxR1 on endogenous GR activity of ORS cells. As anticipated, overexpression of TrxR1 markedly increased endogenous GR activity. These results suggest that decreased TrxR1 may lead to a lower responsivity to glucocorticoid treatment via a decrease in GR activity.

P2

\section{T-Flavanone Suppresses BMP-4 Production in Dermal Papilla Cells}

S. Moriwaki, A. Nagasawa, M. Sasajima, N. Itou,

A. Hachiya, T. Kitahara

Kao Corporation Biological Science Laboratories, Japan

The results from our recent study suggest that there are multiple pathways through which t-flavanone stimulates hair growth. One such pathway previously reported was t-flavanone fs suppression of TGFb2 activation on keratinocytes (10th EHRS, Barcelona, 2003). In this study, we identified another pathway by focusing on dermal papilla cells, which secrete many hair growth-related molecules. We found that conditioned medium collected from dermal papilla cells that had been treated with t-flavanone induced keratinocyte proliferation. Gene Chip and RT-PCR analysis revealed that t-flavanone decreased the mRNA expression of BMP-4 in a dose-dependent manner. The expression and secretion of BMP-4 protein also decreased in a similar manner. Furthermore, the and secretion of BMP-4 protein also decreased in a similar manner. Furthermore, the addition of recombinant BMP-4 to keratinocytes suppressed their proliferation. In contrast, the addition of a BMP-4 neutralizing antibody into an untreated dermal papilla cell-derived conditioned medium promoted keratinocyte proliferation in a dose-dependent manner. These results indicate that t-flavanone stimulates hair growth by suppressing BMP-4 in dermal papilla cells as well as by suppressing activation of TGF-b2 on keratinocytes.

\section{P3}

\section{Induction of Versican by Ascorbic Acid 2-Phosphate in Dermal Papilla Cells}

M.-K. Kim, S.-R. Kim, J.-C. Kim, Y.-K. Sung

Department of Immunology, School of Medicine, Kyungpook National University, Daegu, Korea

We have recently reported that L-ascorbic acid 2-phosphate magnesium salt (Asc 2-P), a derivative of L-ascorbic acid (Vitamin C), promotes the elongation of hair shafts in hair follicles in culture and induces early conversion from a telogen phase to an anagen phase in mice, demonstrating that Asc 2-P affects hair growth and cycling. Here, we investigated whether Asc 2-P regulates expression of versican, which is thought to play an important role in anagen induction and maintenance of normal hair growth, in cultured dermal papilla cells and in dermal papillae of isolated hair follicles in culture. Hair biopsy specimens were obtained from the non-balding occipital scalp region of patients with androgenic alopecia. Dermal papilla cells of the 2 3 passage were used in this study. Immunohistochemical staining of versican was performed with rabbit polyclonal antibody recognizing versican $\mathrm{V} 0$ and $\mathrm{V} 1$ isoforms. We observed that isoforms of versican were up-regulated in dermal papilla cells by Asc 2-P treatment. Immunohistochemical staining also revealed increased expression of versican, at least V0 and V1, in dermal papilla of isolated hair follicles treated with Asc 2-P. LY294002, a pharmacological inhibitor of PI3K, significantly attenuated Asc 2-P-induced versican expression. We also observed that Asc 2-P activates/phosphorylates PKB, a downstream effector of PI3K, and that LY294002 attenuates Asc 2-Pmediated phosphorylation of PKB. In addition, we observed that nuclear $\beta$-catenin is increased by Asc 2-P in a dose-dependent manner and LY294002 reduces nuclear accumulation of $\beta$-catenin. This study demonstrates that Asc 2-P induces versican expression via PI3K signaling and causes nuclear $\beta$-catenin accumulation in dermal papilla cells. Since versican is thought to play an important role in anagen induction and maintenance of the normal hair growth, it would be worthwhile to evaluate vitamin $\mathrm{C}$ or its derivatives as a novel approach for treatment and prevention of hair loss. 
P4

\section{Initial Characterisation of a New Model of Dermal Papilla Cell Culture}

\author{
C. Higgins', G. Richardson', G. Westgate ${ }^{2}$, M. Green ${ }^{3}$, \\ D.J. Tobin ${ }^{4}$, C. Jahoda ${ }^{1}$ \\ ${ }^{1}$ Department of Biological Sciences, Durham University, \\ ${ }^{2}$ Westgate Consultancy, Stevington, Bedfordshire, ${ }^{3}$ Unilever \\ R\&D Colworth, Sharnbrook, Bedford, ${ }^{4}$ School of Life \\ Sciences, University of Bradford, UK
}

Human dermal papilla (DP) cells grown in culture have been studied extensively. However, some key differences between DP cell behaviour in vivo and in culture have been identified. Smooth muscle $\alpha$ actin ( $\alpha \mathrm{SMA})$ is a sheath-cell specific marker in vivo, but once in culture both papilla and sheath cells express $\alpha$ SMA. Cells derived from anagen DP's are highly proliferative whilst the same cells in vivo do not proliferate. Expression of extracellular matrix proteoglycans changes during the hair cycle. The chondroitin proteoglycan Bamacan is expressed in the anagen DP yet lost on entry to catagen and telogen whilst Syndecan-1 is absent in anagen but present in the telogen DP. In contrast, Perlecan expression remains constant throughout the hair cycle. We previously demonstrated that DP cells grown in suspension culture in tiny volumes form small spheroids which appear morphologically to be more akin to DP found in vivo. We have now investigated the differences between the two culture conditions using the expression profile of $\alpha \mathrm{SMA}$, proteoglycans and markers of proliferation. Human DP cells at P4/P5 were plated in $35 \mathrm{~mm}$ dishes or placed in hanging drops. All cells were cultured in MEM containing $10 \%$ FBS. Cells were harvested when $80-90 \%$ confluent whilst spheres were harvested after $30 \mathrm{~h}$ in culture. The two culture methods were compared using RT-PCR and immuno-cytochemistry. The integrity and viability of the spheres was confirmed using TEM and viability markers. Results show that dermal spheres have a different profile from normally cultured DP cells suggesting spheres may be an interesting new model for studying DP cells in vitro. Perlecan and Syndecan-1 expression is similar in both cells and dermal spheres in contrast to Bamacan which is reduced in dermal spheres. $\alpha$ SMA and Ki67 are expressed in DP cells but not in dermal spheres, even though the spheres remained viable.

\section{P5}

\section{Role of Reactive Oxygen Species (ROS) on Androgen-Inducible TGF-Beta 1 Regulation of Dermal Papilla Cells}

H.G. Yoo, Y.J. Kang, S.R. Lee, H.K. Pyo, O.S. Kwon, K.H. Kim, H.C. Eun, K.H. Cho

Department of Dermatology, Seoul National University, College of Medicine, Laboratory of Cutaneous Aging and Hair Research, Clinical Research Institute, Seoul National University Hospital and Institute of Dermatological Science, Seoul National University, Seoul, Korea

Little is known about the roles of androgen on the regulation of redox states in dermal papilla cells, a cellular process known to profoundly increase with aging. The androgen receptor (AR) has been reported to modulate TGF- $\beta 1 / \mathrm{Smad}$ signaling and to be overexpressed in androgen-dependent scalp areas of patients with androgenetic alopecia. The rat vibrissae dermal papilla cell line (DP-6) over-expressed with AR was investigated to evaluate the role of ROS on androgen-induced increase of TGF- $\beta 1$ secretion. AR stablytransfected DP-6 cells were incubated with R1881 or dihydrotestosterone (DHT). Flow cytometry and laser scanning confocal microscopy were undertaken to measure ROS production and an ELISA assay to evaluate TGF- $\beta 1$ secretion after androgen treatment. A TGF- $\beta 1$ promoter activity assay was also performed whether to be influenced by pretreatment of ROS scavengers. Androgen markedly increased ROS generation and the androgen-inducible ROS augmented TGF- $\beta 1$ secretion from dermal papilla cells. Treatment with ROS scavenger or several species of inhibitors decreased ROS production and TGF- $\beta 1$ expression. Luciferase reporter assays showed suppression of TGF- $\beta 1$ promoter signaling by ROS scavengers. In conclusion, our study shows for the first time that androgen-induced TGF- $\beta 1$ accumulation in dermal papilla cells would be mediated by ROS production and prevented by antioxidants or ROS inhibitors.

\section{P6 \\ Study of Cell Senescence in Cultured Primary Balding and Non-Balding Dermal Papilla Cells}

\author{
A.W. Bahta \\ Dermatology (QMUL), London, UK
}

The dermal papilla (DP) expresses androgen receptors and is known to control normal hair growth. The paradox of androgen action in human hair growth is well established but the molecular mechanisms are poorly understood. DP cells derived from frontal (balding) human scalp hair follicles (BDPC) are used to study androgenetic alopecia. Cultured BDPC are known to have a much slower rate of growth in vitro than DP from non-balding sites (NBDPC), however, the cause of this has not been reported. In this study we have investigated the growth of human BDPC and NBDPC in vitro. We observed that BDPC have a limited life span of 2-6 passages. We observed that from passage 2 onwards BDPC but not NBDPC showed a large flattened morphology characteristic of senescent fibroblasts and that once they had assumed this morphology they could no longer be passaged. We showed that these BDPC but not NBDPC of the same passage expressed senescence-associated $\beta$-galactosidase activity at pH-6. Moreover, stress-induced premature senescence was induced with more prominent characteristic behaviour in BDPC than NBDPC after exposure to sub-cytotoxic levels of $\mathrm{H}_{2} \mathrm{O}_{2}$ a known inducer of oxidative stress. Finally BDPC also expressed a wide range of oxidative stress markers including HSP27, Super Oxide Dismutase and Catalase. These data suggest that the well documented, slower in vitro proliferative rate of BDPC is due in part to premature senescence. Moreover, our observation that cultured BDPC express markers of oxidative stress and their response to $\mathrm{H}_{2} \mathrm{O}_{2}$ suggest that oxidative stress may play a major role in male pattern hair loss. Others and we have observed that DHT is able to induce TGF- $\beta 1$ in BDPC. TGF- $\beta 1$ is known to induce oxidative stress and this may therefore, link androgens with oxidative stress and help explain the paradox of androgen action on hair growth. 
P7

\section{Immortalisation and Characterisation of Balding and Non-Balding Dermal Papilla Cell Lines and their Response to Oxidative Stress}

\author{
A.W. Bahta \\ Dermatology (QMUL), London, UK
}

The dermal papilla (DP) expresses androgen receptors and is known to control normal hair growth. The paradox of androgen action in human hair growth is well established but the molecular mechanisms in hair follicles are poorly understood. DP cells derived from frontal (balding) human scalp hair follicles (BDPC) are used to study androgenetic alopecia. However, cultured BDPC are very difficult to obtain, grow very slowly in vitro and have a limited life span of 2-6 passages before they senescence. We have recently shown that BDPC express senescenceassociated $\beta$ galactosidase activity at $\mathrm{pH}-6$. Moreover, stress induced premature senescence was induced with more prominent characteristic behaviour in BDPC than non-balding DPC (NBDPC) after exposure to sub-cytotoxic levels of $\mathrm{H}_{2} \mathrm{O}_{2}$. However, the limited life span of BDPC represents a substantial obstacle for biochemical analysis, genetic manipulation and screens. We therefore, generated immortalised balding and non-balding human DP cells (IBDPC, INBDPC) by ectopic expression of human telomerase. The IBDPC have undergone more than 90 passages without showing any phenotypic changes. As with the primary $\mathrm{DPC}$, the IBDPC maintained their aggregating characteristics and expressed wnt7a, wnt3a, androgen receptor and $5 \alpha$ reductase type 2 mRNA. We established an in vitro co-culture system by growing IBDPC and INBDPC with keratinocytes (KC) to study the androgen effects in hair follicles. Androgen suppressed the growth of $\mathrm{KC}$ when grown with IBDPC and this could be partially reversed using a neutralising antibody to TGF- $\beta 1$. In addition we have also observed that IBDPC retains increased sensitivity towards $\mathrm{H}_{2} \mathrm{O}_{2}$ than INBDPC cell lines. Therefore, immortalised DP cell lines show similar characteristics to primary DPC and they will be of major help to us in our attempt to understand the actions of androgens on hair growth and enable the development of better treatment for androgen dependent hair disorders.

\section{P8 \\ Human Follicular Dermal Papilla/Sheath Express the Genes for Both Soluble and Membrane-Bound Stem Cell Factor, While the Matrix Expresses, C-Kit, its Receptor: Implications for the Control of Hair Pigmentation}

T. Vafaee, I.D. Oliveira, S.M. Picksley, V.A. Randall

Department of Biomedical Sciences, University of Bradford, Bradford, UK

Stem Cell Factor (SCF) or Mast Cell Growth Factor (MGF), Steel factor or c-kit ligand (CL), is a paracrine factor which regulates rodent hair pigmentation. We showed previously that cultured dermal papilla cells secrete SCF and hypothesise that the dermal papilla is a local source of SCF regulating human hair pigmentation. SCF exists in both soluble and plasma membrane-bound forms; it binds to plasma membrane receptor, c-kit. This study was designed to determine where c-kit and SCF are found in human hair follicles. Scalp samples, treated with RNAlater to inhibit mRNA degradation, were microdissected to isolate lower follicles; some hair matrixes and dermal papilla/dermal sheaths were isolated separately. Total and poly(A)RNA were isolated, cDNA prepared and reverse transcription-polymerase chain reactions (RT-PCR) undertaken with primers for c-kit and SCF; cDNA quality was checked using â-actin primers. PCR products were separated by gel electrophoresis, checked for size and sequenced. Immunohistochemistry of frozen scalp sections was also performed to localise c-kit and determine whether its expression mirrored melanocyte marker, Mel-5. Lower follicle samples $(\mathrm{n}=5)$ produced bands for both forms of SCF and ckit. Hair matrix samples $(n=6)$ expressed c-kit and 2 of 3 dermal papilla/sheath extracts expressed both soluble and bound SCF. Sequence analysis showed $100 \%$ correlation with known genes. Immunohistochemistry localised c-kit expression to hair bulb cells, co-localising with Mel-5. Expression of both SCF and c-kit within anagen follicles strongly supports a local paracrine role for this signaling system in human hair pigmentation. The soluble form of SCF detected in whole follicles and the dermal component would correspond to that secreted by cultured dermal papilla cells. C-kit expression by follicles and the matrix components and its co-localisation with melanocyte markers supports a role maintaining melanocyte pigmentation in anagen follicles. The role of the membrane-bound form merits further investigation.

\section{P9}

\section{Hair Growth Modulatory Properties of IL-6 Cytokine Family Members}
K. McElwee ${ }^{1}$, M. Yu ${ }^{1}$, V. Lu ${ }^{1}$, B. Lo ${ }^{1}$, J. Shapiro ${ }^{1}$,
P. Freyschmidt-Paul2, S. Kissling ${ }^{2}$, R. Hoffmann ${ }^{3}$
${ }^{1}$ Department of Dermatology, University of British Columbia, Vancouver, Canada; ${ }^{2}$ Department of Dermatology, Philipp
University, Marburg, ${ }^{3}$ Dermaticum, Freiburg, Germany

The activation of receptor complexes containing glycoprotein 130 (gp130) identifies the IL-6 cytokine family. We examined members of this family, Interleukin-6 (IL-6), interleukin-11 (IL-11), leukemia inhibitory factor (LIF), oncostatin M (OSM), ciliary neurotrophic factor (CNTF), and cardiotrophin-1 (CT-1) in hair follicles. Quantitative RT-PCR using mRNA derived from microdissected, anagen stage, human hair follicles and comparison to non-follicular skin epithelium revealed mRNA for IL-6 and OSM as the most highly expressed while IL-11r, OSMr and CNTFr were the most highly expressed receptors of the evaluated group. Immunohistology suggested expression of IL-6, LIFr and IL-11r in the root sheaths and dermal papilla, while OSM and IL-11, IL-6r and OSMr were expressed in root sheaths alone. CT-1 and LIF demonstrated little if any presence. The gp130 receptor subunit was located in the dermal papilla, root sheaths, hair cuticle and matrix. Human hair follicles from 5 donors were exposed in vitro to $0.1,1,10$, or $100 \mathrm{ng} / \mathrm{ml}$ of OSM or CT-1 for 8 days. Compared to control hair follicles, OSM and CT1 exhibited a growth inhibition capacity. OSM and CT-1 incubated with agarose beads and injected subcutaneously at 1ìg per mouse into telogen skin of mice all 65 days old revealed no capacity to induce anagen hair growth. In contrast, injection of 65 days old mice in which anagen had been induced by hair plucking revealed a hair growth inhibitory capacity for OSM but no significant effect for 
CT1. The data identify IL-6 cytokine family members as modulators of hair follicle growth. High expression of IL-6 cytokine family members can occur as a constituent of skin or hair follicle inflammation such as alopecia areata. In principle, increased expression in cutaneous inflammation may contribute to the promotion of hair loss. The nature of expression distribution circumstantially suggests a role in hair follicle keratinocyte differentiation.

\section{P10 \\ Effect of Substance P on the Mesenchymal - Epithelial Interaction}

L.O. Pi ${ }^{1}$, S.Y. Ahn', J.W. Goo', S.Y. Jeon ${ }^{1}$, T.H. Oh',

S.T. Hwang ${ }^{2}$, W.-S. Lee ${ }^{1}$

${ }^{1}$ Department of Dermatology and Institute of Hair and Cosmetic Medicine, Yonsei University Wonju College of

Medicine, Wonju, ${ }^{2}$ Dr Hwang's Hair Clinic, Seoul, Korea

It has been well known that epithelial - mesenchymal interaction in the hair follicle is crucial for hair growth control. Among constituents of the skin, the hair follicle is an organ with the highest density of nerve fibers. Recently, it has been reported that neuropeptides, which are secreted by nerve fibres, play an important role in hair growth and hair cycle changes. In the previous study conducted by us, we examined mRNAs corresponding to FGF-7, IGF-1, VEGF, IL-1 $\beta$, IL-1 $\alpha$, p53, caspase-3, after culturing hair follicle dermal papilla (DP) cells and outer root sheath (ORS) cells pretreated with substance $\mathrm{P}(\mathrm{SP})$. As results, in DP cell culture, the expression of IL- $1 \beta$ was less than control, but in ORS cells was not shown. From the results, we can suppose signal(s) from the DP cells may influence the ORS cells. In this study, we examined the effect of SP on the mesenchymal - epithelial interaction in the human hair follicle. ORS cells were cultured in only dermal papilla conditioned medium (DPCM) or DPCM treated with SP. Then we observed the expression change of the ORS cell proliferation and gene expression. As results, the proliferation of ORS cells continuously increased when they were cultured in the DPCM treated with SP for $48 \mathrm{~h}$. It may be concluded that various growth factors exist in the DPCM treated with SP, and influenced the proliferation of outer root sheath cells.

\section{P11 \\ Determining the Molecular and Cellular Basis of Hair Follicle Induction}

\section{G. Richardson \\ Durham University, Durham, UK}

The mammalian epidermis and hair follicle (HF) are unique developmental systems characterised by their capacity for continuous renewal (epidermis) and cycling (HF). The HF arises due to a series of reciprocal signaling interactions between the embryonic skin epithelium and mesenchyme. These early events of HF induction take place at around 14.5 days post coitus (dpc) in the mouse, concomitant with the transformation of the simple epithelium to a more differentiated and stratified epithelium. In this study, we performed microarray analysis to study global gene expression changes during epidermal morphogenesis in the mouse at $12 \mathrm{~h}$ intervals between 12.5 and
$15.5 \mathrm{dpc}$. At each time point, we used enzymatic and mechanical techniques to separate the embryonic skin dermis from the epidermis and each RNA sample was hybridised to Affymetrix MOE430A arrays. Our list of significant transcriptional changes has uncovered several potential new players in dermal and HF morphogenesis in addition to corroborating previously identified signaling pathways (Wnt, BMP, Shh, FGF, and EGF). Differential expression throughout the various time points was validated by RT-PCR and expression of selected genes examined at the RNA and protein level by in situ hybridization and immunohistochemistry, respectively. We have been using an embryonic skin organ culture model, to investigate the effects of specific genes on normal hair follicle morphogenesis. The EGFR mediated signaling pathway has previously been demonstrated to be important in HF morphogenesis. Having found differential expression of members of the EGF family we investigated the role of these, and two other genes (Chordin Like 1 and Gremlin both having known roles in BMP signaling) by means of embryonic skin organ culture, to determine their effect on normal hair follicle morphogenesis.

\section{P12}

\section{A Model for Hair Morphogenesis}

\author{
J. Qiao, E. Philips, J. Teumer \\ Intercytex Ltd., Woburn, MA, USA
}

In this study, we have developed a model to examine hair morphogenesis. The assay combines an intact donor epidermis with a suspension of dermal cells, which could be from a variety of donors. The epidermis and the dermal cells are applied to a subcutaneous graft bed created on a dorsal skin flap. When the flap is repositioned over the graft, the graft is in a subcutaneous position such that it is protected after implantation. The assay is simple to perform and overcomes some of the limitations of other assays for hair induction. Using the assay, both freshly isolated and cultured dermal cells as well as cultured dermal papilla cells from several species all induced hair development. The induced hairs were aesthetically indistinguishable from those of the epidermal donor in length, thickness, and pigmentation, and they were also histologically normal. Time course studies show that the process of hair morphogenesis in the grafts was similar to embryonic hair morphogenesis. In addition to serving as a convenient test for the hair inductive capacity of dermal papilla cells, the assay may also be used to examine specific cellular and molecular interactions during hair morphogenesis, such as epithelialmesenchymal interactions and melanogenesis.

\section{P13 \\ Induction of Synapse Associated Protein 102 (SAP 102) Expression in Cyclosporin A-Stimulated Hair Growth}

\author{
J.-K. Park, C.D. Kim, M. Im, Y.-J. Seo \\ Department of Dermatology, Chungnam National University, \\ Daejeon, Korea
}

Cyclosporin A (CsA) has been used as a potent immunosuppressive agent for inhibiting graft rejection after organ transplantation. 
However, CsA provokes lots of side effects including hirsutism, the phenomenon of abnormal hair growth in the body. In this study, we attempted to identify the genes related with CsA-induced hair growth. When topically applied on the back skin of C57BL/6 mice, CsA produced a prominent hair growth stimulating effect. Using differential display reverse transcription-polymerase chain reaction (DD RTPCR) technique, several genes were identified to be differentially expressed by CsA. Among those, the expression of SAP102 was verified using competitive RT-PCR. The result showed that the expression of SAP102 was significantly induced by CsA treatment in the back skin of C57BL/6 mice. However, the increase of SAP102 mRNA was also seen in spontaneous anagen mice, suggesting that induction of SAP102 is one event of the anagen hair growth response regardless of how the growth state was induced. SAP102 was not expressed in cultured human hair outer root sheath and dermal papilla cells. Immunohistochemistry analysis showed that CsA induced the expression of SAP102 in the perifollicular region of mouse anagen hair. These results suggest that SAP102 is induced in the anagen phase of the hair cycle and has an indirect role in hair growth one event of the anagen hair growth response regardless of how the growth state was induced. SAP102 was not expressed in cultured human hair outer root sheath and dermal papilla cells. Immunohistochemistry analysis showed that CsA induced the expression of SAP102 in the perifollicular region of mouse anagen hairs. These results suggest that SAP102 is induced in the anagen phase of the hair cycle and has an indirect role in hair growth.

\section{P14 \\ Topical Administration of Cyanocobalamin (Vitamin B12) Showed Suppression of Potassium Channel Inhibitor (Tolbutamide) and Induction of Murine Hair Anagen Phase and Synergistic Effect with Minoxidil}

N.-H. Park

Skin Research Team, Amore Pacific Corporation R\&D Center, South Korea

Vitamin B12 is well known to be important for growth, cell reproduction, blood formation, and protein and tissue synthesis. Cyanocobalamin and hydroxycobalamin acetate are used to treat pernicious anemia, nutritional vitamin B12 deficiency, malabsorption of vitamin B12, and other cases of vitamin B12 deficiency. We investigated the active effect of vitamin B12 on NIH3T3 fibroblast in ATP-sensitive potassium channel-dependent fashion and on murine anagen indution. The in vitro effects of established potassium channel openers (minoxidil) and inhibitors (tolbutamide), plus vitamin B12 were indirectly assessed on NIH3T3 fibroblast in the absence of aminoglycoside antibiotics and phenol red, and 5\% fetal bovine serumsupplemented medium. Tolbutamide inhibited $28 \%$ proliferation of NH3T3 fibroblast at $2.5 \mathrm{mM}$ concentration as reported previously. When minoxidil and cyanocobalamin was treated to the fibroblast with $2.5 \mathrm{mM}$ tolbutamide, growth inhibition of NIH3T3 fibroblast was suppressed $51.49 \%$ by $100 \mathrm{uM}$ minoxidil $38.39 \%$ by $1 \mathrm{uM}$ cyanocobalamin, respectively. Two topical preparations of single dose of minoxidil (1\%) and cyanocobalamin $(0.03 \%)$ were topically administered with vehicle (ethanol/ propylene glycol/water $=3 / 2 / 5$ ) on clipped dorsal area of female telogen phase $\mathrm{C} 57 \mathrm{bl} / 6$. The grown hair weights of topical $1 \%$ minoxidil preparation (113.14 \pm $11.02 \mathrm{mg}, \mathrm{p}<0.001)$ and $0.03 \%$ cyanocobalamin $(59.56 \pm$ $13.88 \mathrm{mg}, \mathrm{p}<0.05)$ increased significantly than that of vehicle $(18.47 \pm 4.68 \mathrm{mg})$. When topically administered in mixed preparations $(0.5 \%$ minoxidil and $0.03 \%$ cyanocobalamin), the more grown hair weight $(87.00 \pm 8.39 \mathrm{mg}, \mathrm{p}<0.03 \mathrm{t}$-test with $0.05 \%$ minoxidil $)$ by mixed preparation was observed compared to those of single $0.5 \%$ minoxidil preparation $(44.33 \pm 14.96 \mathrm{mg})$ and of vehicle preparation $(22.13 \pm 13.86 \mathrm{mg})$. Topical cyanocobalamin was applied to atopic dermatitis and psoriasis in previous study; our new topical trial of vitamin B12 will be helpful treatment for alopecia as a single dose or coadministration with minoxidil though the precise mechanism was not elucidated.

P15

Towards the Development of a Simplified Long-Term Organ-Culture Method for Human Scalp Skin Under Serum-Free Conditions

\author{
Z. Lu$^{1}$, R. Paus ${ }^{2}$, K. Koslowski ${ }^{3}$ \\ ${ }^{1}$ Department of Dermatology, 2nd Affiliated Hospital, School \\ of Medicine, Zhejiang University, Hangzhou, China; \\ ${ }^{2}$ Department of Dermatology, University Hospital Schleswig- \\ Holstein, University of Luebeck, Luebeck, ${ }^{3}$ Munich, Germany
}

Organ-culture of human scalp skin is usually performed with serum-containing medium, which limits its analytical usefulness. However, without serum, long-term survival of the skin and its appendages is thought to be problematic. Here, we report that intact human scalp skin can be grown at the air/liquid interface in supplemented, serum-free Williams E medium for up to several weeks. Active hair shaft growth was visible until day 16 and was significantly faster than in MEM $+10 \%$ FBS. LDH release into the medium (as an indicator of cell death) revealed a first peak on day 2, stabilised LDH values between day 3 and 12, and a second LDH peak around day 15 (indicating progressive organ decay). By quantitative immunochemistry, proliferating $(\mathrm{Ki}-67+)$ cells could still be observed in the epithelium of hair follicles even on day 12, and day 17 of serum-free skin organ culture. The number of apoptotic (TUNEL+) cells in the skin epithelium rose steadily after day 5 . Before day 12 , Williams E better protected against cell death than MEM $+10 \%$ FBS. Giemsa stains revealed that mature skin mast cells were observed even after 13 days in culture. The percentage of surviving hair follicles with catagen- or telogen-like morphology gradually increased over time, while after 17 days of culture most hair follicles were found to be in telogen. While epidermis and hair follicle epithelium showed increasing atrophy over time, some long term-surviving epithelial islands were found in association with remnants of follicular structures as late as day 88 . These preliminary data suggest that a very simple serumfree organ culture method allows prolonged human skin and hair follicle survival as well as some limited hair follicle cycling in intact skin for more than 2 weeks under well-defined experimental conditions. This pragmatic assay should become a valuable tool for both skin and hair research. 


\section{P16}

Age-Associated Alterations in Human Scalp Hair Follicle Melanocytes - An in Vitro Study

\section{S. Kauser ${ }^{1}$, G. Westgate ${ }^{2}$, M. Green ${ }^{3}$}

${ }^{1}$ Medical Biosciences, School of Life Sciences, University of Bradford, Bradford, 2Westgate Consultancy, Stevington,

Bedfordshire, ${ }^{3}$ Unilever R\&D Colworth, Sharnbrook,

Bedford, UK

Gradual loss of skin pigmentation with age is associated with a slow decrease in epidermal melanocyte numbers. By contrast, loss of melanocytes in ageing hair follicles (HF) is more abrupt, suggesting a different 'melanogenetic clock' in the latter. Hair graying (canities) is thought to result from an increase in reactive oxygen species (ROS)-associated damage and accumulated oxidative stress in HF melanocytes, coupled with an age-related depletion of the HF melanocyte reservoir, and/or defective cell activation/migration during the cyclic repopulation of successive new anagen hair bulbs. To clarify the mechanism of canities we examined age-associated change in HF melanocyte proliferation and in levels of expression of key melanogenic enzymes and catalase in matched epidermal melanocyte (EM) and hair follicle melanocyte (HFM) cultures derived from young, mid-aged and older donors. This study showed that HFM have a greater proliferative potential than matched EM at all ages. By contrast, the age-related decrease in proliferation was greater in HFM than in matched EM. Moreover, while the expression of gp100, Tyrosinase, Tyrosinase-related protein (TRP)-1 and TRP-2 in vitro also decreased with age, the age-associated down-regulation of the former was greater in HFM than in EM. The exception was TRP-2, which was up-regulated in HFM of aged donors. The level of expression of anti-oxidant enzyme catalase was similar in matched HFM and EM from younger donors but showed an age-dependent downregulation in expression. Strikingly, a greater decrease in expression was detected in HFM than in matched EM derived from aged donors. In summary, the age-dependent decrease in melanocyte proliferation in vitro and the differential age-associated down-regulation of melanogenic enzymes in HFM vs. EM suggests that these melanocyte sub-populations are differentially regulated. The decrease in catalase expression with age further suggests that disruption of anti-oxidant defence mechanisms in melanocytes may contribute to loss of pigmentation with age.

\section{P17 \\ Variable Proliferative and Melanogenic Effects of Oestradiol and Selective Oestrogen Receptor Agonists in Human Hair Follicle Melanocytes and Melanoma in Vitro}

\section{A. Meskiri, M.J. Thornton, S. Kauser}

Desmond Tobin Medical Biosciences Research Group, School of Life Sciences, University of Bradford, Bradford, UK

It has long been noted that cutaneous pigmentation can alter during pregnancy and resolve following parturition. Epidemiological studies on malignant melanoma suggest that females may have survival advantage. Moreover, hair color may darken significantly in fair-haired females after puberty. However, despite these clinical observations the role of oestrogens in melanocytic cell biology remains unclear. In this study we examined the effects of oestradiol, and agonists selective for oestrogen receptor (ER)- $\alpha$ and ER- $\beta$ on primary hair follicle melanocytes (HFM) and on melanoma cells with moderate pigmentation (FM55) at concentrations of $10^{-7}, 10^{-8}$ and $10^{-9} \mathrm{M}$. Cells were serum-starved for $24 \mathrm{~h}$, and replenished with $10 \%$ stripped serum (steroid-free) plus oestradiol or the ER agonists for $72 \mathrm{~h}$. The cells were photo-documented for effects on morphology, trypsinised and counted using a haemocytometer, then pelleted for photo-documentation and melanin measurement (at $490 \mathrm{~nm}$ ). Oestradiol and selective agonists to ER- $\alpha$ and ER- $\beta$ reduced HFM proliferation, although little effect was detected morphologically. By contrast, these agents induced a mild to moderate increase in HFM melanin levels. Oestradiol and the ER- $\alpha$ and ER- $\beta$ selective agonists moderately upregulated proliferation in FM55 melanoma, again with little effect detected morphologically. As with HFM, oestradiol and selective agonists to ER- $\alpha$ and ER- $\beta$ induced a mild to moderate increase in FM55 melanoma pigment levels. These results suggest that oestrogens have a similar melanogenic effect on both HFM and FM55 melanoma cells, but these melanocytic cells types exhibit opposite proliferation responses. In summary, these preliminary findings suggest that HFM pigmentation may be regulated by oestrogens acting via ER- $\beta$ and ER- $\alpha$. Agonists at these receptors may also be implicated in regulating cell proliferation differentially in normal (e.g. HFM) and transformed melanocytic cells (melanoma).

\section{P18}

\section{Origins and Importance of 18 Methyl} Eicosanoic Acid (18MEA) in Human Hair

\author{
L. Albiston'², M. Davis', P. Parmar'1, G. Harrap², S. Goff', \\ G. Westgate ${ }^{3}$ \\ ${ }^{1}$ Unilever R\&D Colworth, Sharnbrook, Bedford, ${ }^{2}$ Unilever R\&D \\ Port Sunlight, Bebington, Wirral, 3Westgate Consultancy, \\ Stevington, Bedfordshire, UK
}

Hair fibres contain complex lipid membranes which are likely to confer important properties to the hair fibre but whose organisation and composition is poorly understood. The lipids in the hair fibre originate either from the follicle or are sequestrated from sebum. Differential extraction can be used to resolve the 'free' and 'bound' lipid fractions. Hair contains an unusual branched chain fatty acid, 18MEA, believed to be covalently bound within the cuticle. 18MEA has been described by a number of groups, in particular in relation to absence in the hair from subjects with maple syrup urine disease (MSUD), a defect in the metabolism of branched chain amino acids. In order to study the biosynthesis of integral hair lipids including 18MEA, human hair follicles were isolated from facelift scalp skin and metabolically labelled using 14C-acetate and 14C-isoleucine. The hair fibres grown in vitro were isolated; the covalently bound fatty acids extracted and separated using reversed phase HPLC. Using appropriate standards a biosynthetic profile of hair follicle derived integral fatty acids was determined. The same extraction and analysis methods were used to characterise integral fatty acids in a range of hair samples, including fibres from subjects with MSUD. Furthermore, hair samples from MSUD patients and age/sex/ethnic matched samples 
were examined by TEM to re-examine any cuticular abnormalities associated with MSUD. Results show that $18 \mathrm{MEA}$ is predominantly synthesised from isoleucine, demonstrating the pathway for conferring the c21 methyl branch to the molecule. Hair samples from subjects with MSUD showed a deficiency in 18MEA, but morphologically and ultrastructurally the hair fibres appeared normal. In summary, hair fibres are variable with respect to their overall integral lipid composition which may explain some of the differences in hair fibre properties between individuals. The MSUD results suggest that 18MEA, although unusual, confers no unique ultrastructural feature to hair fibres.

\section{P19}

\section{Characterisation of Liver X Receptors within the Pilo-Sebaceous Unit}

L. Russell, W. Harrison, C.C. Zouboulis, J. Burrin ${ }^{1}$, M.P. Philpott

Centre for Cutaneous Research, London, UK; 'Department of Endocrinology, WHI

Liver X receptors (LXR) play a well documented role in the differentiation and proliferation of human keratinocytes. Currently, little is known of their presence and role in the human pilosebaceous unit. Here, we have shown that LXRs are present in numerous cell types derived from the human pilosebaceous unit. Specifically, we used RTPCR to locate the mRNA of both LXR $\alpha$ and $\beta$ within not only primary keratinocytes but also within outer root sheath keratinocytes, dermal fibroblasts, connective tissue sheath fibroblast and dermal papilla fibroblasts as well as within the immmortalised keratinocyte cells lines HaCaT and N/TERT and immortalised sebocyte cell line SZ95 [1]. Western blot analysis was used to identify the proteins of both isoforms within each of these cell types. This was further confirmed by immunohistochemistry using monoclonal and polyclonal LXR antibodies on human hair-bearing skin. We also identified previously unreported functional roles for LXR within human skin. By stimulating SZ95 cells with synthetic LXR agonists TO901317 and GW683965 for 2 days we were able to stimulate lipogenesis. Using the 2-14C-labelled sodium acetate incorporation assay enabled us to quantify the stimulatory effects of these treatments, revealing a significant increase in lipogenesis compared to vehicle treated control cells, indicating an increase in the rate of differentiation. The antiproliferative qualities of LXR stimulation have been indicated by the treatment of primary keratinocytes, N/TERT and SZ95 with synthetic LXR agonists for 2 and 5 days in vitro. Using the MTT assay a highly significant decrease in cell number was observed in agonist treated cells compared to vehicle treated controls. The effects of LXR agonists acting specificity on functioning endogenous receptors was confirmed using the LXRE-LUC reporter assay. This study has not only elucidated the previously unknown presence of LXR with the pilosebaceous unit but additionally highlighted potential therapeutic targets within the field of acne.

\section{Reference}

1 Zouboulis et al: J Invest Dermatol 1999;113:1011-1020.

\section{P20}

\section{Severe Alopecia Areata is Associated with a Functional Polymorphism in the PTPN22} Gene

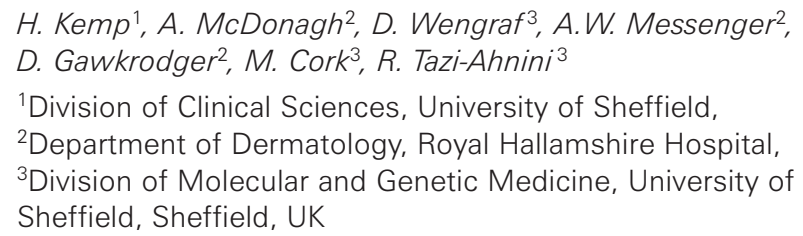

Recently, the non-synonymous C1858T substitution in the PTPN22 gene, which encodes lymphoid protein tyrosine phosphatase (LYP), has been shown to be associated with susceptibility to a range of autoimmune disorders including vitiligo, type I diabetes mellitus and rheumatoid arthritis. LYP has an important role in negative control of T-cell activation and in T-cell development and is therefore a plausible candidate for involvement in the pathogenesis of alopecia areata (AA). The C1858T substitution gives rise to an amino acid change from Arg to Trp at position 620. The objective of this study was to determine whether the disease-associated PTPN22 1858T (W620) allele was also associated with AA. The allelic distribution of the PTPN22 C1858T alleles was determined in 196 English patients with AA and 507 ethnically matched control subjects. The frequency of the $1858 \mathrm{~T}$ allele did not differ significantly between the AA patients and the control group. In the cases, out of a total of 392 alleles, 41 (10.5\%) encoded the W620 variant compared to 86 of $1,014(8.5 \%)$ in the control group. However, in patients with severe disease, 25/168 $(14.9 \%)$ alleles were $1,858 \mathrm{~T}$ and this frequency differed from that in the control group $(p=0.0127)$. These results suggest that the LYP non-synonymous R620W substitution may have an influence on the severity of AA and provide further evidence for autoimmunity as an aetiological factor in this condition.

\section{P21}

\section{Phenotypic and Molecular Characterisation of the Depilated Hairloss Mutation}

\author{
A.W. Lee1, I. Smyth², R. Porter ${ }^{3}$, I. Jackson'1, Y. Gondo 4 , \\ L. Mckie \\ ${ }^{1} \mathrm{MRC}$ Human Genetics Unit, Edinburgh, ${ }^{2}$ Keratinocyte \\ Laboratory, CRUK London Research Institute, London, \\ ${ }^{3}$ Department of Dermatology, University of Cardiff, UK \\ ${ }^{4}$ Functional Genomics Research, RIKEN GSC, Kanagawa, \\ Japan
}

Depilated (dep) is an autosomal recessive mutation that is characterised by the loss of hair shortly after birth. Mutant mice can be identified by an unusually thin and short hair coat as early as a week of age when hairs first emerge from follicles. Adult hairs are greasy, matted and slightly depigmented. Previous studies using recombination assays have shown that dep is a defect of hair follicles due to deficiency of the epidermis rather than the dermis. Skin sections at successive timepoints reveal acanthosis, intense pigment clumps in anagen, and dilated follicles in telogen which might promote the observed premature entry into 3rd anagen. Dep has been mapped by complementation 
analysis to a region of $160 \mathrm{~kb}$ on mouse chromosome 4, within which Zdhhc21 is the most probable candidate based on sequencing data and published literatures that exclude the other 2 genes within the interval. Sequencing of Zdhhc21 in dep reveals a single amino acid deletion at residue 233 which is predicted to be intracellular. Zdhhc21 is a transmembrane zinc finger protein with DHHC-CRD (Asp-His-His-Cyscysteine-rich domain) that belongs to a 23 -member family. The function of Zdhhc21 is currently unknown but DHHC-CRD is hypothesised to be a palmitoyltransferase domain. To prove that Zdhhc21 is the gene responsible for dep, we are using transgenic rescue with a mouse BAC. To generate additional mutant alleles of Zdhhc21, a genebased screen is being performed on archives of DNA samples from individual F1 ENU-mutagenised mice generated at the MRC Mammalian Genetics Unit and the RIKEN GSC respectively. Currently, we have isolated one mutation from the MRC archive which converts leucine to phenylalanine at a residue that lies immediately upstream of DHHC-CRD (L91F). Our RT-PCR data suggests that Zdhhc21 is ubiquitously expressed; whole-mount in-situ assay is being carried out to finely assess its expression pattern at embryonic stages.

\section{P22}

A Study on the Effect of Genetic Variants of the Autoimmune Regulator Promoter, on AIRE Activity and Downstream AIRE-Regulated Genes

\author{
T. Lovewell1, M. Cork ${ }^{1}, 2$, D. Wengraf 1 , A.W. Messenger ${ }^{2}$, \\ A. McDonagh ${ }^{2}$, R. Tazi-Ahnini ${ }^{1,2}$ \\ ${ }^{1}$ Division of Genomic Medicine, University of Sheffield, \\ 2Dermatology Immunogenetics Group, University of \\ Sheffield, Royal Hallamshire Hospital, Sheffield, UK
}

Alopecia areata has been found to occur $\sim 40$ times more commonly and with greater severity amongst Autoimmune Polyglandular Syndrome-1 (APS-1) patient populations. APS-1 is a monogenic disease, caused by loss of function mutations in the Autoimmune Regulator (AIRE) gene. The AIRE gene is primarily expressed in the thymus and is believed to modulate promiscuous gene expression, which in turn, seems to be involved in T-cell negative selection. It has been shown that the function of AIRE is dose dependent, thus we intend to investigate whether natural polymorphisms in the AIRE promoter region affect the level of AIRE transcription. We used WAVE denaturing HPLC to screen the first $591 \mathrm{bp}$ upstream of the AIRE transcription start site from 32 alopecia areata patients and 32 control samples. Using the MatInspector 2.1 and the rVista 2.0 programmes we have investigated the predicted binding sites of AIRE protein to the promoters of the human and mouse homologues of casein $\alpha \mathrm{S} 1$, keratin 10 and keratin 12 . We identified the $-103 \mathrm{C} / \mathrm{T}$ SNP, found within the minimal promoter region, and the $-528 \mathrm{G} / \mathrm{A}$ SNP, found upstream of the minimal promoter region. We cloned the $-528 \mathrm{G} /$ $-103 \mathrm{C},-528 \mathrm{G} /-103 \mathrm{~T}$ and $-528 \mathrm{~A} /-103 \mathrm{C}$ promoter haplotypes upstream of a firefly luciferase reporter gene. We are currently performing reporter gene assays in thymic cells TEC $1 \mathrm{~A} 3$ in order to measure the efficiency of the different promoter variants. Binding sites were identified in the analysed promoters and were conserved between mouse and human. We have cloned these promoters into the pCR2.1 vector, and are currently using DNase I protection assays and electrophoretic mobility shift assays to investigate the physical binding of AIRE protein to these promoters. Our main aim is on one hand to analyse the effect of SNPs on the AIRE promoter activity and on the other hand to dissect the mechanisms by which AIRE regulates gene expression.

\section{P23 \\ Comparison of mRNA Expression in Hair Follicles of Hairless with Normal Rats}

\author{
S. Tani
}

Pharmacology, Josai University, Japan

The hairless rat is a mutant of Wistar rats with short, fine and scattered hairs. Now many hair growth factors were reported. We compared the expression of hair growth factor mRNAs in hair follicles of hairless with normal rats. RT-PCR was performed using extracts of RNA from hair follicles of hairless and normal Wistar rats. Real time PCR was carried out for quantitative measurement of the mRNA. Amounts of BMP3-, BMP7-, TGF- $\beta-$, IGF1-, IGF2-, and EGFmRNA in hair follicles were almost equal in hairless and normal rats. However, BMP receptor IA is less expressed in hairless rats than normal rats. Amounts of IGF1 and IGF2 receptor-mRNA were almost equal in both rats. HGF-, FGF5-, VEGF- and ephrin A3 receptormRNAs were not detected. $\beta$-actin was less expressed in hair follicles of the hairless rat. Amounts of BMP receptor IA-mRNA in bone were equal in hairless and normal rats. Also, amounts of $\beta$-actin-mRNA in the dermal tissue were almost equal. These results suggested that BMP receptor IA and $\beta$-actin were less expressed in hair follicles of hairless rat specifically. On quantitative analysis by real time PCR, amounts of BMP receptor IA-mRNA and $\beta$-actin were about $1 / 3$ and $1 / 2$, respectively. It was reported that the hair follicle and IRS were reduced in BMP receptor IA KO mice. These results show BMP receptor IA was one of the important factors on hair growth. At the same time, a specific function of $\beta$-actin in hair growth is presumed. In conclusion, the hairless rat is a useful animal for the development of hair restorations, e.g. a stimulator of BMP receptor IA or $\beta$-actin in hair follicles might be a kind of hair restorative.

\section{P24 \\ Human Hair Follicles Express Genes for all Components of Potassium Channels, Enabling Direct Responses to Minoxidil and Other Types of Potassium Channel Regulators}

K. Shorter, S.M. Picksley, V.A. Randall

Department of Biomedical Sciences, University of Bradford, Bradford, UK

How minoxidil, a widely used treatment for hair loss, actually acts is not well understood. Suggested mechanisms include stimulation of the vasculature or ATP-sensitive potassium (KATP) channels within the follicle itself. KATP channels consist of inwardly rectifying $\mathrm{K}^{+}$ 
channel (Kir) and regulatory sulfonylurea receptors (SUR). Although deer follicles are directly regulated by potassium channel regulators in vitro, human studies are predominantly negative. This study was designed to determine whether genes for both specific SUR, (SUR1, SUR2A, SUR2B) and the actual pore channel protein itself (Kir6.2) are expressed in human follicles. Human scalp was treated with RNAlater ${ }^{\mathrm{TM}}$ stabilisation reagent to preserve mRNA. Anagen follicles were isolated by micro-dissection, pooled and total RNA extracted. RNA quality was checked before poly(A)RNA isolation; DNase treatment removed any contaminating DNA, before cDNA synthesis by reverse transcription. Reverse transcription-polymerase chain reaction (RT-PCR) was carried out using primer sets designed to detect Kir6.2, SUR1, SUR2A and SUR2B. cDNA quality was assessed by RT-PCR using primers for the highly expressed gene, $\beta$-actin. PCR products were separated by gel electrophoresis and appropriately sized bands sequenced. $\beta$-actin was expressed in all 5 samples, confirming cDNA quality. Bands were produced which correlated to Kir6.2, SUR1 and SUR2B; no bands corresponding to SUR2A were detected. Sequence analysis of all products confirmed homology to the known human gene sequences. These results confirm for the first time that human hair follicles express the genes for all components of KATP channels; the Kir6.2 channel protein and two different SUR: SUR1 and SUR2B. The SUR2B form, previously detected in human cultured dermal papilla cells, would facilitate hair growth stimulation by minoxidil; however, the SUR1 form would not respond to minoxidil and has not previously been reported in human follicles. Further studies of these channels should lead to novel treatments for hair loss.

\section{P25 \\ Novel Compound Heterozygous Nonsense Mutations in the Hairless Gene Causing Atrichia with Papular Lesions}

\author{
G. Ashoor ${ }^{1}$, R. Greenstein'2, H. Lam³ , A. Martinez-Mir 3 , \\ A. Zlotogorski ${ }^{4}$ A. Christiano ${ }^{5}$ \\ 'St John's Institute of Dermatology, The Guy's, King's \\ College and St Thomas Medical School, London, UK; \\ ${ }^{2}$ Division of Human Genetics, University of Connecticut \\ Health Center, Connecticut, ${ }^{3}$ Department of Dermatology, \\ Columbia University, College of Physicians and Surgeons, \\ New York, USA; " ${ }^{2}$ epartment of Dermatology, Hadassah- \\ Hebrew University Medical Center, Jerusalem, Israel; \\ ${ }^{5}$ Department of Genetics and Development, Columbia \\ University, College of Physicians and Surgeons, New York, \\ USA
}

Atrichia with papular lesions (APL) is a rare autosomal recessive condition resulting from mutations in the hairless (HR) gene. In the present study, we investigated the molecular basis of APL in a nonconsanguineous Korean family. Direct automated DNA sequencing of the HR gene and restriction digestion analysis were used to identify and confirm the mutation in our proband. Sequencing of the HR gene revealed two novel nonsense mutations in exons 2 and 4, which were subsequently confirmed via enzymatic restriction. No mutations have previously been detected in this population. The growing number of heterozygous mutations in non-consanguineous pedigrees supports the hypothesis that APL is more common than previously expected.

\section{P26}

Experiences of Persons with Alopecia Areata

S. McHale ${ }^{1}$, N. Hunt ${ }^{2}$, N. Coulson ${ }^{2}$

${ }^{1}$ Department of Psychology, Hallam University, Sheffield,

2IWHO, University of Nottingham, Nottingham, UK

Alopecia is a chronic inflammatory dermatological disease that affects the hair follicles. It manifests in varying degrees of severity from small patches of hair loss to total loss of all head hair through to the severest form where all body hair is lost. There are few physical symptoms apart from, e.g., discomfort caused by the loss of eyebrows and eyelashes. Research into the psychological impact of alopecia has been limited, and is usually secondary to medical research, but it demonstrates that there are psychological problems associated with the disorder (Hunt and McHale, 2005), including anxiety and depression. We present the findings from two qualitative studies into the psychological impact of alopecia. We recruited 162 participants to generate spontaneous written accounts and a further 34 participants who took part in an e-mail interview over several days. Accounts, which varied in length from a few lines of text to 6 pages, were subjected to analysis using a grounded approach. Findings from both studies indicated similar degrees of psychological distress and focussed on negative personal, social and medical effects. Successful coping was discussed by very few respondents, but included behavioural change and acceptance of appearance and identity change. Psychological distress was not related to the severity of hair loss. It is important to highlight the psychosocial consequences to physicians, and to provide alopecia sufferers with the means to deal with the psychological sequelae of hair loss.

\section{P27}

The Lucy's Pelt. When Men Became Hairless and How He Managed to Survive

\section{A. Rebora \\ University of Genoa, Genoa, Italy}

Why, of the 193 species of primates, man alone looks glabrous remains a mystery, but the real problem is when the mutation occurred and how hominids managed to survive a substantially deleterious mutation. According to the available evidence, the hairless mutation occurred before the Pan-Homo divergence. Denudation however must have become evident much later. Fur, in fact, was necessary when Australopithecinae moved to the hot savannah where hairlessness would have left them defenceless against the African sun. Furthermore, traces of Homo erectus were found in Caucasian Georgia where the average winter temperature is $0^{\circ} \mathrm{C}$. As, at that time, Homo erectus did not control fire, he could not survive hairless. Homo erectus was hairy, therefore, though probably less hairy than his progenitors. To reconcile an early mutation with a late denudation, we must accept that mutations display their phenotypic effects also progressively. The runaway sexual selection model provides a plausible explanation. 


\section{P28}

\section{Ethnic Characteristics of the Eyelashes: Comparative Analysis in Asian and Caucasian Females}

\author{
O.S. Kwon', J.I. Na', B.J. Kim', W.S. Park', J.K. Oh, \\ K.H. Cho ${ }^{1}$, H.C. Eun ${ }^{1}$, K.H. Kim ${ }^{1}$ \\ ${ }^{1}$ Department of Dermatology, Seoul National University \\ Hospital, Seoul, ${ }^{2}$ Skin Research Institute, AmorePacific R\&D \\ Center, Gyeonggi-do, ${ }^{3}$ Rich Hair Clinic, Seoul, South Korea
}

Despite the similar biochemical composition of human hair between races, physico-morphological characteristics are not identical in different ethnic groups. Eyelashes have been far less investigated than scalp hair, and the information available is insufficient. This study was performed to obtain basic information about eyelashes and to clarify differences between female eyelashes in Asians and Caucasians. Twenty Asian and 10 white Caucasian female volunteers aged between 20 and 29 years were recruited. Lateral digital photographs of upper eyelashes were taken and curl-up and lift-up angles of upper eyelashes were measured from lateral views. Images of upper eyelashes were also taken by using a phototrichogram technique and total numbers, lengths, and thicknesses of upper eyelashes were obtained. The central portion of eyelashes or whole eyelashes were clipped and images were taken immediately and 7 days later to obtain the growth rate and anagen ratio of upper eyelashes. Numbers and thickness of eyelash cuticular layers were obtained by electron microscopy. Compared with Caucasians, Asian eyelashes revealed lower lift-up and curl-up angles, lower numbers, and a thicker transverse diameter. But, no statistical difference was observed in length or growth rate. Duration of anagen was estimated at about 2 months. The eyelash anagen ratio obtained from 5 Asians was $17.8 \pm 3.3 \%$. By electron microscopy, the number of cuticular layers in transverse section was greater in Asian $(8.0 \pm 1.2)$ than Caucasian females $(6.5 \pm 1.1)$, but no statistical difference was found in single cuticle layer thickness between two groups. Moreover, eyelash characteristics were not influenced by eye makeup in either race. Our results on eyelash morphology and growth characteristics demonstrated significant ethnical differences in Asian and Caucasian females that could provide basic information for future investigations.

\section{P29}

\section{The Morphology of Scalp Whorls and Anterior Hairlines in Koreans}

\section{J. Yoo, C. Kim, T. Park \\ Department of Dermatology, Kangbuk Samsung Hospital, Korea}

Although scalp whorls and the anterior hairline are important parts for the morphology of the scalp hair pattern, only a few studies on them have been reported. This study was performed to obtain data about the classification and incidence of various scalp whorl patterns, the average heights of the anterior hairlines, and the types of anterior hairlines and their incidence. We evaluated the number, direction and location of the parietal scalp whorls, measured the heights of the frontal and temporal hairlines according to the 5 standard lines, and identified the shape of the anterior hairlines for 484 men and 171 women. Of the 655 subjects, $89.6 \%$ had single parietal scalp whorls, while $6.9 \%$ had double whorls. The single clockwise type was more common than the single counterclockwise types, and the locations of the single whorls were in middle, right and left, in decreasing order of frequency. Of the double whorls, the cases of the same direction were more common than those of the opposite direction. The average heights of the anterior hairlines were higher for men than for women. For the classification of the anterior hairline, the linear type was the most common for men and the round type was more common for women. We described the present results as a guideline for the further study of hair-related abnormalities and also for the esthetic design of hair transplantation.

\section{P30}

\section{Scleroderma En Coup De Sabre with Secondary Pseudo-Cicatricial Alopecia and Partial Loss of Eyebrow and Eyelashes}

\section{A. Finner, J. Shapiro}

Department of Dermatology and Skin Science, University of British Columbia, Vancouver, Canada

We report a 44-year-old caucasian male who developed linear progressive hair loss and a skin-coloured depression of his right scalp and forehead over the last 8 years with partial loss of the right eyebrow and upper eyelashes. The eyelids appear normal. There is no history of previous trauma or associated symptoms. Histology of a scalp biopsy is consistent with morphea and shows a normal pattern on elastic staining. ANA was negative. A neurological examination was normal. Scleroderma en coup de sabre (LSCS) is regarded as a variant of morphea and can cause secondary cicatricial alopecia. Because end-stage lesions show a widened hyalinised dermis and preserved elastic fibres, the condition has been categorised as pseudo-cicatricial. Autoimmunity is thought to be involved in the etiology of morphea. Displacement, local inflammation or cytokines may cause the permanent alopecia. While loss of eyebrows is not uncommon in LCSC, involvement of eyelashes has only been reported once before. A close association between LSCS and Progressive Facial Hemiatrophy (PFH, Parry-Romberg Syndrome) has been suggested, with PFH being a deeper variant of LSCS with neurologic and ophthalmologic manifestations. In PFH, mosaicism or a dysregulation of the sympathetic nerves has been hypothesised (trophoneurosis). The latter is supported by PFH development after sympathectomy and the distribution along the branches of the trigeminal nerve, which is accompanied by sympathetic fibres. Hair follicles are surrounded by a network of nerves. We therefore hypothesise that the loss of eyelashes could also be related to alterations in piloneural interaction. In summary, LCSC can cause secondary pseudo-cicatricial alopecia. The pathogenesis of this condition and which factors lead to loss of the relatively distant eyelashes in this case are unclear. 


\section{P31}

\section{The New Classification of Androgenetic Alopecia}

\author{
W.-S. Lee', B.I. Ro ${ }^{2}$, S.P. Hong ${ }^{1}$, The Korean Hair Research \\ Society Group ${ }^{3}$ \\ 'Department of Dermatology and Institute of Hair and \\ Cosmetic Medicine, Yonsei University Wonju College of \\ Medicine, Wonju, ${ }^{2}$ Department of Dermatology, College of \\ Medicine, Chung-Ang University, Seoul, ${ }^{3}$ Thirteen \\ Dermatologic Centers, Korea
}

Androgenetic alopecia is considered to be the most prevalent type of hair loss occurring after the age of puberty in humans. The disorder can be classified by several patterns of hair loss. Now, the HamiltonNorwood classification system in men and the Ludwig grade system in women are commonly used to describe the pattern. But it has been thought that these existing classifications have some limitations. The classification methods are too detailed and complicated and less stepwise, so that it is difficult to be used actually. Since the most subjects were Caucasians in their publications, they cannot suit Korean men with androgenetic alopecia, who characteristically tend to show a more female pattern of hair thinning than Caucasians. So the existing methods cannot classify all types in all races. In addition, clinicians must use a different system by gender to classify the pattern. Therefore, we designed to develop a new classification system which is easily available and comprehensive, regardless of race and sex, and actually to apply the new system to classify the types of androgenetic alopecia. Between November 2004 and June 2005 in 13 dermatologic centers in Korea, a total of 2,213 Koreans with androgenetic alopecia (1,768 men and 445 women) were analyzed according to sex, age, basic and special type (reflecting alopecia pattern), based on the new classification system. The basic types, representing the shape and degree of anterior hair line, were divided into 4 types (Linear, M-/C-/U-shape). The special types, represent the female pattern $(\mathrm{F})$ and the density of hair on the vertex $(\mathrm{V})$. We can decide on the final type of a subject after putting together both types. According to these classification principles, type MF in men and type LF in women are the most common.

\section{P32 \\ The Efficacy of $5 \%$ Minoxidil vs. Combined Topical Agent of $5 \%$ Minoxidil and $0.01 \%$ Tretinoin on Male Pattern Hair Loss: A Randomised Comparative Clinical Trial}

\section{H.-S. Shin \\ Department of Dermatology, Seoul National University \\ College of Medicine, Seoul, Korea}

Topical minoxidil 5\% solution has been widely used to stimulate new hair growth and to help stop the hair loss successfully in men with male pattern hair loss (MPHL). Nevertheless, it is not easy for the patients to keep applying the solution twice a day regularly. The purpose of this 18-week, double-blind, randomised study was to compare the efficacy and safety of the treatment of combined solution of $5 \%$ minoxidil and $0.01 \%$ tretinoin once a day with conventional topical 5\% minoxidil therapy applied twice a day in the treatment of
MPHL. A total of 29 male patients (25-50 years old) with MPHL (Hamilton type III-V) were randomly assigned to apply 5\% minoxidil twice daily or the combined agent once a day at night using vehicle placebo in the morning. The efficacy parameters were (1) changes in total hair count, mean hair thickness and linear growth rate measured by phototrichogram and (2) the patient and investigator subjective assessment of change in scalp coverage. After 18-weeks of therapy, significant increases in the all macrophotographic variables of total hair count, mean hair diameter and linear growth rate were shown in both treatment groups. Moreover, the 5\% minoxidil and the combined agent groups also demonstrated similar improvement in the subjective global assessment by patient and investigator. There was no statistically significant difference between the treatment groups. The occurrence of side effects like pruritus or local irritation was similar in $5 \%$ minoxidil group ( 5 of 14 subjects) and the combined agent group (5 of 15 subjects). In conclusion, the efficacy and safety of combined $5 \%$ minoxidil and $0.01 \%$ tretinoin once-daily treatment would be equivalent to the conventional $5 \%$ minoxidil twice-a-day therapy for treatment of MPHL.

\section{P33 \\ Eyebrow Transplantation in a Case of Keratosis Pilaris Atrophicans}

N. Farjo, B. Farjo

Farjo Medical Centre, Manchester, UK

Eyebrow transplantation is a well-recognised restorative procedure in cases of diminished/absent eyebrow hair. The commonly treated causes include traction loss (i.e. eyebrow plucking), traumatic loss (burns, accident scars), and congenital fine/sparse eyebrows. Hair transplantation in scarring alopecia only is successful in cases of 'burnt-out' conditions and then only in situations where there is no compromise to the blood supply. The case described is that of a 33year-old man who presented with longstanding problems with his eyebrows beginning in infancy. His eyebrows were largely absent and there was erythema and 'pimples' of the skin in the upper canthal area. He had not previously sought a medical opinion on his condition so the patient was sent to a dermatologist for a diagnosis. The dermatologist's opinion was that this was a case of burnt-out keratosis pilaris atrophicans/ulerythema oophyrogenes. Keratosis pilaris atrophicans is a condition present from early infancy and is characterised by erythema and horny plugs beginning in the outer halves of the eyebrows and then moving medially. It may affect the cheeks also. The eyebrows are eventually destroyed. There is no reference in the literature of a case that has been transplanted and as the patient was keen to have a trial of eyebrow restoration the decision was made to perform a single procedure. Bilateral single-haired grafting was performed with incisions made by 15 degree sharpoint blades. A total of 100 hairs per eyebrow were inserted. During the procedure there was higher than average bleeding and swelling was noted to be high on the left side. The grafts in this area were also more difficult to place. After approximately 3 months the patient noticed new hairs growing. At the postoperative review at 6 months, scalp hairs were growing abundantly. The patient was happy with the results and opted for two further procedures to increase the density. Two years after the initial procedure the hairs are growing and the skin has shown no signs of inflammation. 


\section{P34}

\section{8-nm Excimer Laser Treatment of Lichen Planopilaris of the Scalp}

B.P. Vavricka, S. Haug, I. Eliades, R. Trüeb

Department of Dermatology, University Hospital Zurich, Zurich, Switzerland

Lichen planopilaris (LPP) is a chronic inflammatory disorder eventually leading to permanent scarring alopecia. Therapy of LPP is difficult, though corticosteroids, antimalarials, retinoids, and immunosuppressive agents have been reported with variable success. Photochemotherapy with topical psoralen and UV-A represents a treatment with comparable results, less toxicity, but it is costly, time consuming and messy. The handheld XTRAC excimer laser (monochromatic $308 \mathrm{~nm}$ UVB-light), allows selective treatment of lesional skin with higher single doses, fewer treatments, and lesser cumulative doses. It has been proven to be effective in other chronic inflammatory skin disorders. The aim of the study was to evaluate the efficacy of XTRAC excimer laser for treatment of LPP and LPP variants (frontal fibrosing alopecia). Subjects were scheduled to receive 8-24 treatments. Thirteen patients ( 11 female, 2 male) with biopsy-proven LPP, unresponsive to conventional therapies, were included. Laser radiation was delivered twice weekly to one half of the scalp, the untreated served as control. A treatment protocol analogous to that for psoriasis was chosen. The scalp area was assessed weekly, with respect to erythema, infiltration, hyperkeratosis, hair growth, and pigmentation. Patients were asked to rate the severity of their baseline symptoms itch, pain, and hair loss. The patients received a mean dose of $4,538.5 \mathrm{~mJ} / \mathrm{cm}^{2}$ after an average of 10 treatments. In 6 patients there was no difference between the two sides. Four patients initially showed improvement of erythema, but after 4 weeks of treatment there was no difference. One patient had sustained improvement of pruritus and erythema. Two patients had increased hair growth, one with repigmentation of hair. New lesions appeared to be more responsive than old lesions. We conclude that the XTRAC 308-nm excimer laser represents a potential treatment modality for LPP. Probably, patient selection and treatment protocol need to be improved for superior results, since only 3 of 13 patients had a benefit.

\section{P35}

\section{Marginal Fibrosing Alopecia, Fibrosing Alopecia in a Pattern Distribution and Central Scarring Alopecia: One New and Two Known Variants of Circumscribed Lichen Planopilaris}

\section{A. Finner, J. Shapiro \\ Department of Dermatology and Skin Science, University of British Columbia, Vancouver, Canada}

We report three cases of primary lymphocytic cicatricial alopecia due to Lichen planopilaris (LPP) in non-reticulated, circumscribed locations. The first case is a 63-year-old Caucasian postmenopausal woman who developed a receding atrophic irregular frontal, temporal and occipital hairline with eyebrow thinning. The second case is a 25year-old Caucasian male presenting with scarring alopecia confined to the fronto-parietal and vertex scalp, which is also affected by pattern hair loss (PHL). The third case is a 40-year-old Caucasian female with an expanding scarred alopecic patch in the vertex. In all 3 patients, adjacent hair-bearing areas showed clinical and histological features of LPP. The lesions were itchy and there were no signs of lichen planus of other cutaneous sites, mucous membrane or nails. This new variant of LPP, which we call Marginal Fibrosing Alopecia, not only affects the frontal and temporal hairline as previously described in Postmenopausal Frontal Fibrosing Alopecia (PFFA), but also the occipital hairline. As in cases of PFFA, there is eyebrow thinning and the condition is clinically and histologically indistinguishable from multifocal LPP, except for the special location. The second phenotype resembles a variant previously described as fibrosing alopecia in a pattern distribution. Its relation to PHL has not been clearly defined. The third case represents a central morphologic variant of LPP, which is commonly observed by us and other authors. The central scalp appears to be a generally more susceptible area for primary scarring alopecias. Marginal fibrosing alopecia is a new variant of circumscribed LPP not previously described. Other variants present as frontal fibrosing alopecia, fibrosing alopecia in a pattern distribution and central scarring alopecia. The significance and reasons for the preference of certain scalp regions in these subsets of LPP and in cicatricial alopecia in general have yet to be elucidated.

\section{P36}

\section{Clinical Studies in Hair Transplantation Using Digital Imaging}

A. Finner ${ }^{1}$, K. McElwee ${ }^{1}$, D. Zloty ${ }^{1}$, R. Hoffmann' ${ }^{2}$, J. Shapiro ${ }^{1}$

${ }^{1}$ Department of Dermatology and Skin Science, University of British Columbia, Vancouver, Canada; ${ }^{2}$ Dermaticum, Freiburg, Germany

Hair transplantation (HT) is the most successful treatment option in androgenetic alopecia (pattern hair loss) with recent advancements in technique. But quantitative evidence based on data on regrowth is still lacking. This is partly due to general difficulties when assessing hair growth. Unique problems in HT studies include variations in technique, inadequate sample size, incomplete follow-up and a lack of reproducible methods. Trichoscan ${ }^{\circledR}$ (TS) is a recently developed tool to measure hair growth parameters. For TS, a magnified photo of a circular target area of $1.8 \mathrm{~cm}^{2}$ is entered into a digital imaging software to calculate hair growth parameters. For repeated measurements, the target area is either marked by a pin-point temporary tattoo or an angioma is used as a landmark. A pilot study was initiated to observe hair density in a recipient target area before and after HT. The number of hairs transplanted into the target area is counted. TS is performed before the procedure and after 2 weeks, 4 and 6 months. TS is a practical method to assess hair density and has received good patient feedback. Exact data collection is crucial, as slight changes in pigment, hair length and location can influence results. Results show a partial initial decrease in hair density and thicker, denser hair on follow-up visits. The initial decrease is most likely due to shock loss, which has never been documented before. From digital imaging techniques, such as TS, we expect new insights into the dynamics of hair growth following HT and preassessment of donor area density for improved planning of procedures. With further improvement, computerised methods may eventually play an important role in evaluations of different HT techniques. 


\section{P37}

\section{An Evaluation of Hair Dermatoscopy in Assessment of Hair Shaft Abnormalities}

\author{
M. Wallace, D. de Berker \\ Bristol Dermatology Department, Bristol Royal Infirmary, \\ Bristol, UK
}

Examination of hair contributes diagnostic information in a range of adult and paediatric conditions. We have set out to examine the relative merits of transmitted light microscopy and Dermatoscopy in hair shaft microscopy. Typical examples of selected conditions were identified from an extensive collection of scalp hair and examined using a Dermlite Pro HR II dermatoscope and Nikon E200 light microscope, with paired cross-polarising filters. Hair shaft characteristics were photographed using a Nikon Coolpix 4500 digital camera. Dermatoscopy was helpful in detecting tapered hairs, weathering, monilethrix, pediculosis capitis, peripilar casts, exclamation mark hairs of alopecia areata, bubble hair and pili torti. It was less helpful in pili annulati and unhelpful in detecting 'tiger-tail' banding in trichothiodystrophy. Light microscopy provided greater detail in almost all cases. It was necessary for detection of hair cuticle changes and added significantly more information in detecting characteristic features of trichothiodystrophy, pili annulati, bubble hair and pili torti. Dermatoscopy is a useful clinical tool when examining numerous hair conditions. It is most revealing in conditions manifested by gross changes in shaft outline and colour, where reflected light is valuable. It is not helpful for detection of features within the shaft or at higher levels of resolution. When added to its ability to aid evaluation of scalp surface charcacteristics, dermatoscopy provides an excellent first-line method of assessment of problems in the hair clinic. Use in vivo allows it to be used for screening of areas of scalp in order to select hairs of greatest diagnostic yield for further assessment. In some instances, it may obviate the need for obtaining a hair specimen. Where detailed or cortical hair shaft features need assessment, transmitted light microscopy remains the standard tool.

\section{P38}

\section{Combined Systemic Therapy with Cyclosporine A and Methyl-Prednisolone in Patients with Extensive Alopecia Areata}

\author{
C.-H. Huh, S.W. Min, C.W. Choi, B.J. Kim', S.W. Youn, \\ K.C. Park \\ Dermatology, Seoul National University Bundang Hospital, \\ 'Dong-Guk University International Hospital, Gyeonggi-do, \\ Korea
}

Alopecia areata infrequently manifests a refractory course and difficulty in treatment. Although its precise pathogenesis is unclear, Tcell-mediated immunological abnormalities have recently been suggested. Cyclosporine A (CsA) is an immunosuppressant originally applied in organ recipients. Nowadays, it is being used in many dermatological conditions such as psoriasis and atopic dermatitis. Hypertrichosis, one of the common side effects of CsA, induced many physicians to prescribe it to treat alopecia areata but its efficacy is still under debate. Therefore, there is no standardised protocol of
CsA in alopecia areata yet. We have adapted combined systemic therapy with CsA (Cipol-N, ChongKunDang, Korea) and methylprednisolone (mPd, Methyron, KunWha Pharmaceutical Co., Korea) on 46 cases with extensive alopecia areata. The starting doses of CsA were varied from $2.5 \mathrm{mg} / \mathrm{Kg}$ to $4 \mathrm{mg} / \mathrm{Kg}$ and $\mathrm{mPd}$, from 0.3 to $0.4 \mathrm{mg} / \mathrm{Kg}$ ( $4 \mathrm{mg} \mathrm{mPd}=5 \mathrm{mg}$ prednisone). After $\mathrm{mPd}$ was gradually tapered first, CsA was tapered next. Among 46 volunteers, 43 completed the whole schedule while 3 patients dropped out due to sideeffects of combination therapy (gastrointestinal disturbance and facial oedema). Among 43 cases, 5 cases did not show remarkable improvements and the overall success rate was $88 \%$ (38/43). Combined systemic therapy with $\mathrm{CsA}$ and $\mathrm{mPd}$ may be an effective treatment modality in patients with extensive alopecia areata but close monitoring of side-effects is required.

P39

Trichoadenoma of Nikolowsky

F. Mardones, A. Cardemil, H. Fuenzalida, N. Navarrete,

F. Schröeder

Department of Dermatology, University of Chile, Santiago, Chile

Trichoadenoma of Nikolowsky is a rare, benign and slowly growing tumor with follicular differentiation. It affects adults, mostly in the face or buttocks where it may be present as a solitary nodular or cystlike lesion. We report the case of a 44-year-old female who consulted for a 2-month-old rapidly growing lesion in the left retroauricular region. Clinically, she had a $2-3 \mathrm{~cm}$ plaque made up of multiple flesh and yellow coloured 3-4 mm papules. Some of the lesions resembled open comedones. The diagnosis of an adnexal tumor was initially proposed, and an incisional biopsy was carried out. Histologically, the tumor was located in the dermis and was made up of multiple and different sized infundibular cysts filled with keratin. Extended strands of basoloid cells were present connecting the cysts. A sparse surrounding stroma was also observed. These findings were consistent with the diagnosis of trichoadenoma of Nikolowsky. Finally, curettage and electrosurgery of the remaining lesion was performed, with good postoperative results. Trichoadenoma of Nikolowsky was first described in 1958 and very few cases are described in the international literature. To our knowledge, this is the first reported case in Chile.

P40

\section{The Role of Iron and Lysine in Women with Increased Hair Shedding and Low Serum Ferritin: A Randomised Controlled Trial}

\section{P. Birch, A.W. Messenger, D.H. Rushton}

Department of Dermatology, Royal Hallamshire Hospital, Sheffield, 'School of Pharmacy and Biomedical Sciences, Portsmouth, UK

The role of iron stores in female hair loss is controversial. Some have claimed that low iron stores contribute to both chronic telogen effluvium and female pattern hair loss, but others dispute the idea. We have performed a double-blind randomised controlled clinical trial 
comparing the response to oral iron supplement and placebo in women with low iron stores complaining of excessive hair shedding. Entry criteria included: complaint of excessive hair shedding for at least 6 months without visible reduction in hair density; serum ferritin $<70 \mathrm{ug} / \mathrm{l}$. Subjects were randomised to take an oral product containing ferrous glycine sulphate and 1-lysine $(96 \mathrm{mg}$ elemental iron, $2 \mathrm{~g}$ 1-lysine per day) or placebo for 36 weeks. Trichograms were performed at 0 and 36 weeks. Serum ferritin was measured at 12 weekly intervals. We recruited 29 subjects of whom 21 completed the trial (10 active, 11 placebo). There was no significant difference in any baseline variable between the study groups. There was a significant increase in serum ferritin (week 0: $32.1 \mu \mathrm{g} / 1 \pm 17.2$; week 36: $100.8 \pm 52.9 ; \mathrm{p}<0.001$ ), and a significant decrease in the telogen $\%$ (week 0: $15.6 \pm 5.2$; week 36: $12.1 \pm 5.1 ; \mathrm{p}<0.05)$ in subjects taking the active treatment. No change in serum ferritin (week 0: $30.1 \pm 15.6$; week 36 : $33.0 \pm 15.3$ ) or telogen \% (week 0: $13.2 \pm 5.8$; week $36: 12.5 \pm 5.5$ ) was found in those taking placebo. While we were able to show within group changes, we were unable to demonstrate that increasing iron stores had any effect on anagen:telogen ratios between groups of women with chronic telogen effluvium. Excessive hair shedding may have diverse aetiologies and it is possible that iron deficiency is the cause in some women but our results indicate that a larger study group would be needed to demonstrate a conclusive effect.

\section{P41}

\section{Hair Restoration and Anti-Gray Hair Effects by Topical Roxithromycin}

T. Ito, N. Ito, N. Seo, H. Yagi, H. Hashizume

Masahiro Takigawa Dermatology, Hamamatsu University School of Medicine, Hamamatsu, Japan

There are still few effective topical substances for androgenetic alopecia (AGA). In this study, we found that roxithromycin (RXM) has anti-apoptotic and anti-gray hair effects on AGA in vitro, and indeed, clinically improved or prevented hair loss of AGA. RXM is a 14-member new macrolide antibiotic and has been extensively used for prevention and treatment of systemic and local infection. In addition, recent studies revealed RXM also has various modulatory bioactivities to many kinds of cells, such as Langerhans cells, keratinocytes and $\mathrm{T}$ cells. Human hair follicles, obtained from plastic surgery with informed consent, were cultured for 6 days. In the control group, hair elongation was $1.96 \pm 0.19 \mathrm{~mm}$. On the other hand, it was $3.4 \pm 0.20 \mathrm{~mm}$ and $3.32 \pm 0.19 \mathrm{~mm}$ in $5 \mu \mathrm{M}$ RXM and $10 \mu \mathrm{M}$ RXM treated group, respectively in vitro. The groups treated with $5 \mu \mathrm{M}$ CLM $(2.17 \pm 0.13 \mathrm{~mm}), 10 \mu \mathrm{M}(1.93 \pm 0.18 \mathrm{~mm}), 5 \mu \mathrm{M}$ EM $(2.85 \pm 0.13 \mathrm{~mm}), 10 \mu \mathrm{M}$ EM $(2.05 \pm 0.19 \mathrm{~mm})$ showed no significant hair elongation compared with the control group. Next, 11 male individuals suffering from AGA topically applied $8 \mathrm{ml} \mathrm{RXM}$ at $0.5 \%$ in alcoholic solution once a day for 6 months with informed consent. Photographs were then scored, 4 patients apparently showed improvement of hair restoration, 4 patients were more than slight improvement, and 3 patients were no improvement. Topical RXM used under these conditions was safe without any local and systemic adverse effects. The beneficial effects of RXM seem to result from prevention of apoptosis of matrix cells and melanocytes in human hair follicles. RXM is a promising candidate for effective and safe treatment of AGA.

\section{P42}

\section{Hair Loss of Type Telogen Effluvium and Androgenetic Alopecia in Women is Not Correlated to Low Zinc Levels in the Serum}

\author{
R. Lurie', U. Ron'², M. David ${ }^{3}$, E. Hodak ${ }^{3}$
}

${ }^{1}$ Maccabi Healthcare Services, Pediatric Dermatology Hair

Clinic, Schneider Children's Medical Center of Israel,

2Department of Biochemistry, The George S. Wise Faculty of

Life Sciences, Tel Aviv University, ${ }^{3}$ Department of

Dermatology, Rabin Medical Center, Beilinson Hospital,

Sackler Faculty of Medicine, Tel Aviv University, Israel

Medical literature as to the correlation between hair loss and various nutritional deficiencies in general and specifically zinc is scarce. There is no well-based study which established that in people with no background diseases, linked to known nutritional deficiencies, hair loss was caused by a zinc deficiency. Nevertheless, the popular view is that low serum zinc concentrations cause hair loss, and many trichologists, dermatologists or medical doctors carry out serum zincconcentration tests, and prescribe zinc supplements as treatment for hair-loss. Our goal was testing whether common types of hair loss in women, i.e. Telogen Effluvium (TE), Chronic Telogen Effluvium (CTE) and Androgenetic Alopecia (AGA) are correlated to low serum zinc concentrations. The study included 1,459 women (mean age: 38.5 years, range: $17-89$ years) who visited a dermatology clinic which specialises in the diagnosis and treatment of hair-loss. The diagnosis was based on a full medical anamnesis accompanied by a physical examination. 765 women were reported as suffering from TE; 645 suffered from AGA; and 49 women suffered from CTE. In some unclear cases the diagnosis was based upon a trichogram examination using the German method. Serum zinc concentrations were tested after an overnight fast and in a single laboratory. The patients did not use nutritional supplements at least 3 months prior to the blood test. Serum zinc concentrations measured were normal in all women (mean: $108.1 \mu \mathrm{g} / \mathrm{dl}$; the norm ranges between $55-165 \mu \mathrm{g} / \mathrm{dl}$ ), except for 2 women, whose serum zinc levels were slightly under the norm $(53,54)$. In conclusion, hair loss of types, TE, CTE and AGA in women is not linked to low serum zinc concentrations. Hence, in our opinion, testing for serum zinc levels should not be a routine examination for diagnosing hair loss in women.

\section{P43}

\section{Assessment of Safety and Efficacy of Topical Photodynamic Therapy Using 20\% 5-AIA on Excessive Hair}

\section{J. Varghese, A. Anstey, S.Varma, N. Nicolau \\ University of Wales College of Medicine, Cardiff, UK}

The aim of this study was to examine the safety and efficacy of topical 5-ALA PDT in hirsutism. To achieve this, 13 subjects were recruited in a single blind study; nine were healthy volunteers and four were hirsute patients. Two matching test areas measuring $4 \mathrm{~cm}^{2}$ of normal hair was selected on the forearm in healthy volunteers. However in patients with hirsutism two areas of excessive hair measuring $4 \mathrm{~cm}^{2}$ were selected. In both the patients and the healthy 
volunteers, one of the selected areas was treated with $20 \%$ topical 5-ALA and other area with placebo cream. Hair counting using a video microscope was carried out before and after treatment in both the treated areas. Treated areas were also photographed in a standardised manner before and after treatment. One area of excessive hair was treated with PDT using topical 5-ALA and other sites were treated with topical placebo cream. Subjects treated with 5-ALA sites showed a mean hair loss of $44 \pm 25$ by week one compared with the control sites $16 \pm 16$. This difference was statistically significant $(\mathrm{p}=0.003)$. The mean hair loss in the treated site by week four was $57 \pm 20 \%$ in comparison the placebo site showed a mean hair loss of $29 \pm 22$. This difference failed to reach statistical significance $(p=0.06)$. Erythema and pain as assessed by a clinical scoring grade (0-3) according to severity was experienced by all the subjects on the day of treatment but improved by week one and totally disappeared by week four on both the treated site and placebo site. We have concluded that topical PDT using 20\% 5-ALA is an effective and cheap therapeutic option for patients with hirsutism. As side-effects associated with the treatment are tolerable and reversible, PDT also appears suitable for treatment of larger areas of excessive hair.

\section{P44}

\section{Evaluation of Hair Transplantation into Various Recipient Sites: Lower Leg, Nape of the Neck, Palm, Hand Dorsum, Lower Back and Wrist}

\author{
S.T. Hwang \\ Dr. Hwang's Hair Clinic, Seoul, Korea
}

In 1998, scalp hairs were transplanted to the lower leg. Three years later, some of the scalp hairs previously transplanted on the lower leg were re-transplanted to the nape of the neck near the occipital scalp. Hair transplantation to the palm, hand dorsum, lower back was done in 2001. We reported different hair growth characteristics according to the recipient site. The purpose of this study is to evaluate whether the transplanted hairs show different growth characteristics during long-term follow-up. The survival rate, growth rate and hair diameter were measured at the 8 years post surgery. The results showed:

(1) The survival rate and growth rate of the transplanted hairs is influenced by the recipient site.

(2) The cycles of the transplanted hairs may change according to the recipient area. rather than being fixed by the internal clock of hair follicles.

(3) The hair growth rate may change immediately after transplantation according to the recipient site and is maintained afterwards.

(4) The volume of transplanted hair follicles may not change regardless of the recipient site.

(5) Skin thickness and/or blood vascularity play a role in hair growth and survival.

\section{P45}

\section{Improvement of Hair Properties by Treatment with Hydrophobic Recognition Domains}

\author{
C.J. Silva, A. Vasconcelos, A. Cavaco-Paulo \\ Department of Textile Engineering, Minho University, \\ Guimarães, Portugal
}

Exposure of the hair to sun, wind and modern hair styling products and techniques (e.g. shampooing, bleaching, dyeing and shaping of hair with wave preparations) imparts significant and unwanted damage to the cuticle and cortex of the hair shaft. This damage results in a loss in body, lustre, and smooth texture as well in increased electrostatic charge, reduced maximum tensile strength, breaking of the hair and in a poor appearance of hairstyles. The use of proteins for cosmetic purposes began more than 50 years ago. The efficacy and substantivity of proteins have been documented by many different methods - both scientific and subjective. In this study, a new methodology of hair treatment is presented by using hydrophobic recognition domains (HRD) that are capable of increasing hair elongation, tensile strength and body, while preventing its damage. The hair samples were treated with HRD in buffer. The tensile strength measurements were done with an Instron 4505 with a load cell of maximum capacity of $2.5 \mathrm{~N}$. Hair cross-sections were analysed by a transmission optic microscope (Olympus BH2) with a magnification of 40X. Also, a panel of 5 technicians evaluated the handle and appearance of the hair. Tensile strength resistance increased about $2 \%$ for hair that was not chemically treated and about $55 \%$ for hair that was previously treated chemically. Interestingly, there was also an increase in the elongation properties of hair (of $6 \%$ for non treated hair and of $15 \%$ for chemically treated hair). Also, by means of transversal cuts, penetration of the HRD was analysed. It was seen, by fluorescence microscopy, that different HRD were penetrating completely inside hair cortex, while others were retained at the surface of hair, in the cuticle layer. This last set of HRD had a higher protecting effect on hair. The results revealed that hydrophobic recognition domains can enhance the elasticity and tensile strength of hair, especially chemically treated hair. In addition, HRD were found to extend the gloss, brightness and the body of the hair.

\section{P46 \\ Ultraviolet Light Induced Hair Shaft Damage after Hair Dyeing}

S.Y. Jeon, L. Q. Pi, W.-S. Lee

Department of Dermatology and Institute of Hair and Cosmetic Medicine, Yonsei University Wonju College of

Medicine, Wonju, Korea

Human scalp hairs protect scalp skin from sunlight, and eyebrows and eyelashes keep our eyes from solar damage and sweat. Also, human hairs have important meaning related to body image in social life. Entering the modern society, industries related with hair care and hair health have been developed continuously. Accordingly, there have been increasing needs about scientific research of hair. The causes of external hair shaft damage are various, and roughly can be 
grouped as physical causes and chemical causes. Physical causes are mainly composed of heat stimuli during artificial hair drying and exposure to sunlight. On the other hand, chemical agents for permanent waving, bleaching and dyeing are well known causes of chemical hair damage. In our previous study, the hair dyeing process induced hair cuticle detachment and intercellular lipid layer damage, and those conditions seemed to recover gradually after 1 week. Among the various causes of damage, sunlight, especially ultraviolet light induced hair damages are inescapable during daily life. Many scientists have studied about photoaging in skin, but concerns about the effects of ultraviolet light to hair have been emerging recently. Hairs exposed to ultraviolet light tend to be dry, coarse and stiff, and usually lose their strength, color and lustre. These photochemical changes are mainly known to come from damage to hair proteins and melanins. Particularly, ultraviolet light of $254-400 \mathrm{~nm}$ can damage hair proteins by oxidation of sulfur-containing molecules on the hair shaft. In this study, we investigated the patterns of morphological and moleculobiological changes from artificial ultraviolet light-induced photo-damaged hairs after hair dyeing by scanning and transmission electron microscopy and hair protein analysis. In results, hair soluble protein changes were induced mainly after UVA radiation, while structural changes such as hair cuticle damage were induced mainly after UVB radiation.

\section{P47 \\ Extrinsic Hair Shaft Damage and Restoration: Implications for Integral Hair Lipid, Hair Soluble Protein and Hair Water Content}

\author{
W.-S. Lee ${ }^{1}$, S.Y. Jeon', Y. Kim², L. Q. Pi ${ }^{1}$, K.M. Lee ${ }^{2}$, I.S. Cho ${ }^{2}$ \\ ${ }^{1}$ Department of Dermatology and Institute of Hair and \\ Cosmetic Medicine, Yonsei University, Wonju College of \\ Medicine, Wonju, ${ }^{2}$ Central Research Laboratory, Aekyung \\ Co., Ltd. Daejeon, Korea
}

The human hair shaft is constantly subjected to repeated environmental assaults, commonly termed 'weathering', which are aggravated by sunlight and exogenous chemical damage such as hair dye or permanent wave. These factors cause extrinsic hair shaft damage in addition to natural intrinsic hair shaft aging. Hair shafts damaged from acute extrinsic assault can be restored to the predamaged state after a substantial period of time. On the hair surface, cells are covered with a thin lipid layer covalently bonded to hair proteins. This integral hair lipid plays a key role in the maintenance of hair integrity including hydrophobicity and stiffness, serving as 'hair barrier'. The hair shafts were treated with various kinds of extrinsic assaults including cosmetic hair dyeing, bleaching, permanent waving, natural sunlight and artificial ultraviolet damage. Scanning and transmission electron microscopy was performed to observe the ultrastructure of the hair shaft and integral hair lipid using a specialised electron microscopic fixative designed by us. In addition, we extracted and concentrated the whole and partial hair soluble protein, then performed electrophoresis and western blot analysis of the hair soluble protein which is released after hair damage. Also, we measured the water content of the hair shaft. From the above experimental procedure, it was discovered that the integral hair lipids were mainly distributed within the intercellular layers of cuticle cells and partly affected by extrinsic hair damages. Also the general architecture of the hair shafts was partly changed by various extrinsic damages and restored to predamaged state. Changes of hair soluble protein and hair shaft water content were also noted.

\section{P48}

\section{Comparison of Microscopic Images of Human and Mammalian Hair Shafts Using Hard X-Ray Microscopy}

S.P. Hong, S.Y. Jeon, T.H. Oh, J.W. Goo, S.Y. Ahn, H.S. Yoon',

W.-S. Lee

Department of Dermatology and Institute of Hair and Cosmetic Medicine, Yonsei University, Wonju College of

Medicine, Wonju, 'Pohang Accelerator Laboratory, Pohang

University of Science and Technology, Pohang, Korea

There have been used various methods of hair investigation. Among them, scanning and transmission electron microscopy have been widely used in the field of ultrastructural study of hair. But, because these methods need sample processing procedures and inevitably devitalise samples, these are of limited value. Recently, it was introduced that microscope using highly coherent, bright third generation X-ray could be an alternative way to visualise the ultrastucture of material. The hard X-ray from the third generation synchrotron light source has a higher energy with better penetration and there is no need for sample preparation so it is more suitable for biological samples without processing artifacts or damage to samples. We investigated the ultrastructure of human and some mammalian hair shafts, including dog, cat, mouse, cow, rabbit and rat. Three distinct structures (medulla, cortex, and cuticular layer) of hair shaft were clearly distinguished in all species except cat. We also found that the detailed characters of each sample were different. Among them, the most distinct characteristic is the cortex structures.

\section{P49 \\ Effects of Eucalyptus Extract on Hair Properties and their Molecular Mechanisms}

\author{
H. Taguchi, S. Ueda, C. Ogawa, H. Yoshida, S. Moriwaki, \\ T. Kitahara
}

Biological Science Laboratories, KAO Corporation, Japan

Characterization and modification of physicochemical properties of hair are essential to develop cosmetic products and advance the biological and cosmetic hair sciences. We previously demonstrated that a long-term topical application of Eucalyptus extract on human scalp has improved physical factors, which determine the appearance, elasticity, lustre, bounce and manageability of hair. However, the mechanism of action remained unclear. In this study, the molecular mechanisms underlying these effects of Eucalyptus extract were examined in vivo and in vitro. Ten healthy volunteers participated in the 3-week study. Half of the scalp was treated with a lotion containing 3\% Eucalyptus extract and the other half was treated with a placebo lotion containing 
all of the test lotion except Eucalyptus extract. Anagen hairs were plucked from both test-sites of the scalp before and after the 3-week treatment. The transcriptional profiles of the hair root cells were compared using DNA microarray hybridization and quantitative RT-PCR. The effect of Eucalyptus extract was also examined in human cultured keratinocytes and dermal papilla cells to find putative genes responsible for improving hair properties. In hair root cells treated with Eucalyptus extract, genes related to the structural constituent, such as filaggrin, S100A8, desmoplakin and epiplakin1 were down-regulated, whereas VEGF and KRTAP4-12 genes were up-regulated. In human cultured keratinocytes, it was confirmed that Eucalyptus extract upregulated VEGF gene expression and induced VEGF protein secretion. Furthermore, VEGF was found to have a role to decrease desmoplakin gene expression in human cultured keratinocytes. These results lead us to predict the involvement of VEGF and desmoplakin in the effect of Eucalyptus extract on hair properties.

\section{P50}

Quantitative Analysis of the Characteristics between Normal Controls and Androgenetic Alopecia in Koreans Using the Computerized Hand-Held Phototrichogram System

\author{
C.H. Huh' ${ }^{1}$, B.J. Kim², J.Y. Kim³ ${ }^{3}$ S.W. Youn ${ }^{1}$, K.C. Park' , B.I. Ro ${ }^{3}$ \\ ${ }^{1}$ Department of Dermatology, Seoul National University, \\ Bundang Hospital, Bundang, ${ }^{2}$ Dongguk University \\ International Hospital, Ilsan, Gyeonggido, ${ }^{3}$ College of \\ Medicine, Chung Ang University, Seoul, Korea
}

The purpose of this study was to probe the effectiveness of the computerized hand-held phototrichogram system, which is easy to use, by comparing the characteristics of hair between a group of Korean men with androgenetic alopecia and a group of men without hair loss. Scalps from 15 normal healthy volunteers and 12 androgenetic alopecia patients were evaluated by the computerized hand-held phototrichogram system (Folliscope, LeadM Corporation, Seoul, Korea). The phototrichogram system was applied to $15 \mathrm{~cm}$ above glabella of the volunteers for the baseline measurement after shaving and marking with a tattoo to ensure reproducibility. Two days later, hair density, linear hair growth rate, and hair thickness were automatically calculated immediately after applied again to the same site. Our results showed differences in hair density $\left(88.6 / \mathrm{cm}^{2}\right.$ in androgenetic alopecia, $155 / \mathrm{cm}^{2}$ in normal), linear hair growth rate $(141 \mu \mathrm{m} /$ day in androgenetic alopecia, $198 \mu \mathrm{m} /$ day in normal), and hair thickness (38.8 $\mu \mathrm{m}$ in androgenetic alopecia, $71 \mu \mathrm{m}$ in normal) between the two groups. We can easily obtain the quantitative data of the hair of normal controls and androgenetic alopecia patients in Koreans using the computerized hand-held phototrichogram system. This showed lower hair density, thinner hair diameter, slower hair growth rate in the Korean androgenetic alopecia group than in the non-hair loss group. The ease of measurement can make phototrichogram suitable for clinical practice as well as in research.

\section{P51}

\section{Caffeine Counteracts the Growth Inhibitory Effect of Testosterone in Ex Vivo Human Hair Follicles from Male Patients with AGA \\ T.W. Fischer ${ }^{1,2}$, U.C. Hipler ${ }^{1}$, P. Elsner ${ }^{1}$ \\ ${ }^{1}$ Department of Dermatology and Allergology, Friedrich- Schiller-University, Jena, ${ }^{2}$ Department of Dermatology and Venerology, University Hospital Schleswig-Holstein, University of Lübeck, Lübeck, Germany}

Androgenetic alopecia (AGA) is a common problem in men of all ages, starting at the age of 20 and affecting about $50 \%$ at the age of 50 . The underlying cause is an androgen-dependent miniaturization of genetically predetermined hair follicles, modulated by dihydrotestosterone (DHT) and the expression of androgen receptor (AR). Here, we used ex vivo hair follicles from balding areas of men with AGA and cultivated them in vitro to investigate the effects of testosterone and caffeine, the latter being a promising candidate for hair growth stimulation. Hair follicles from 14 biopsies, taken from the vertex areas from male AGA patients, were cultivated for $120-192 \mathrm{~h}$ in the presence of normal William's E medium (control) or William's E medium containing different concentrations of testosterone and/or caffeine. The outcome parameters were hair shaft elongation and keratinocyte proliferation assessed by Ki-67 staining of longitudinal hair follicle cryosections. Testosterone which is metabolized by intrafollicular 5 - $\alpha$-reductase to DHT lead to significant growth suppression at the concentration of $5 \mu \mathrm{g} / \mathrm{ml}$. This suppression was neutralized by caffeine in concentrations of 0.001 and $0.005 \%$. Moreover, caffeine alone lead to a significant stimulation of hair follicle growth compared to normal medium. The results were confirmed by proliferation assessment with Ki-67 staining. Androgen-dependent growth inhibition of ex vivo hair follicles from patients suffering from AGA was present in the human hair organ culture model. This model imitates the clinical situation of AGA in vitro and may serve for future studies to screen new substances against androgen-dependent hair loss. Caffeine counteracted the inhibitory effect of testosterone and was identified as a stimulator of human hair growth in vitro, a fact which may have important impact on clinical management of AGA. 
Aassi, B. 62

Adekunle, S. 58, 61

Ahn, S.Y. 66, 78

Albiston, L. 68

Anstey, A. 76

Asaad, K. 58, 61

Ashoor, G. 71

Assouly, P. 59

Atoyan, R. 57

Autran, B. 59

Bahta, A.W. 64, 65

Birch, P. 75

Blount, M. 56

Blume-Peytavi U. 59

Boehm, M. 60

Botchkarev, V. 57

Bull, J.J. 57

Burrin, J. 69

Cardemil, A. 75

Carpenter, E. 60

Cavaco-Paulo, A. 77

Cho, I.S. 78

Cho, K.H. 64, 72

Choi, C.W. 75

Choi, D.-K. 63

Christiano, A. 71

Chung, B.C. 58

Combadiere, B. 59

Cork, M. 61, 69, 70

Coulson, N. 71

David, M. 76

Davis, M. 68

de Berker, D. 75

Eliades, I. 74

Elsner, P. 79

Eun, H.C. 64,72

Farjo, B. 73

Farjo, N. 73

Fessing, M. 57

Finner, A. 72, 74

Fischer, T.W. 79

Freyschmidt-Paul, P. 62, 65

Fuenzalida, H. 75

Gawkrodger, D. 69

Gilhar, A. 62

Gilkes, J.J.H. 59

Goff, S. 56, 68

Gondo, Y. 69

Goo, J.W. 66, 78

Goyal, S.P. 58

Green, M. 64, 68

Greenstein, R. 71

Gupta, P. 62

Hachiya, A. 63

Hadam, S. 59
Hamazaki, T.S. 56

Harrap, G. 68

Harrison, W. 57, 69

Hashizume, H. 76

Hasnaoui, I.R. 59

Haug, S. 74

Higgins, C. 64

Hipler, U.C. 79

Hodak, E. 76

Hoffmann, R. 65, 74

Hong, S.P. 73,78

Huh, C.-H. 75, 79

Hummel, S. 62

Hunt, N. 71

Huq, S. 58, 61

Hwang, S.T. 66, 77

Im, M. 63, 66

Ito, N. 76

Ito, T. 76

Itou, N. 63

Iwabuchi, T. 56

Jackson, I. 69

Jahoda, C. 64

Jang, S. 63

Jeon, S.Y. $66,77,78$

Jouanique, C. 59

Kalish, R.S. 62

Kang, Y.J. 64

Karoo, R. 61

Kaufman, G. 62

Kauser, S. 68

Kemp, H. 69

Kim, B.J. 72, 75, 79

Kim, C. 72

Kim, C.D. 63,66

Kim, J.-C. 58, 63

Kim, J.Y. 79

Kim, K.H. 63, 64, 72

Kim, M.-K. 58, 63

Kim, K.M. 58

Kim, S.-R. 63

Kim, Y. 78

Kishimoto, J. 56

Kissling, S. 65

Kitahara, T. 63, 78

Koslowski, K. 67

Kwack, M.-H. 58

Kwon, O.S. 64, 72

Lademann, J. 59

Lam, H. 71

Lee, A.W. 69

Lee, J.-H. 63

Lee, K.M. 78

Lee, S.R. 64

Lee W.-S. 66, 73, 77, 78

Lew, B.-L. 58

Lo, B. 65
Lovewell, T. 61, 70

Lu, V. 65

Lu, Z. 67

Lurie, R. 76

Lyonnet, C. 59

Mahe, B. 59

Manning, J. 55

Mardariev, A. 57

Mardones, F. 75

Margot, Z. 62

Martinez-Mir, A. 71

Matard, B. 59

McDonagh, A. 61, 69, 70

McElwee, K. 65, 74

McHale, S. 71

Mckie, L. 69

Meskiri, A. 68

Messenger, A.W. 54, 61, 69, 70,75

Min, S.W. 75

Moriwaki, S. 63, 78

Na, J.I. 72

Nagasawa, A. 63

Navarrete, N. 75

Nelson, L. 58

Nicolau, N. 76

Ogawa, C. 78

Oh, J.K. 72

Oh, T.H. 66,78

Okochi, H. 56

Oliveira, I.D. 65

Osada, A. 56

Papageorgiou, N. 60

Park J.-K. 63, 66

Park K.C. 75,79

Park N.-H. 67

Park, T. 72

Park, W.S. 72

Parmar, P. 56, 68

Paus, R. 67

Philips, E. 56, 66

Philpott, M.P. 57, 69

Pi, L.Q. 66, 77, 78

Picksley, S.M. 65, 70

Porter, R. 69

Pyo, H.K. 64

Qiao, J. 56, 66

Ramsay, I.D. 59

Randall, V.A. 65,70

Rebora, A. 71

Reygagne, P. 59

Richardson, G. 64, 66

Ro, B.I. 73,79

Ron, U. 76

Rushton, D.H. 59, 75
Russell, L. 69

Ryu, H.K. 58

Sahajpal, V. 58

Sasajima, M. 63

Schaefer, H. 59

Schröeder, F. 75

Seo, N. 76

Seo, Y.-J. 63, 66

Shapiro, J. $65,72,74$

Sharov, A. 57

Sharova, T. 57

Sharpe, D. 58, 61

Shin, H.-S. 73

Shorter, K. 70

Silva, C.J. 77

Sim, W.-Y. 58

Slusareewicz, P. 56

Smyth, I. 69

Sterry, W. 59

Stevenson, S. 58, 61

Stieler, K.M. 59

Sung, Y.-K. 58, 63

Taguchi, H. 78

Tani, S. 70

Tazi-Ahnini, R. 61, 69, 70

The Korean Hair Reserch Society Group 73

Teumer, J. 56, 66

Thornton, M.J. 58, 68

Tobin, D.J. 60, 61, 64

Trüeb, R. 74

Ueda, S. 78

Vafaee, T. 65

Varghese, J. 76

Varma, S. 76

Vasconcelos, A. 77

Vavricka, B.P. 74

Vitacolonna, M. 62

Vogt, A. 59

Wallace, M. 75

Watt F.M. 55

Wengraf, D. $61,69,70$

Westgate, G. 56, 64, 68

Yagi, H. 76

Yoo, H.G. 64

Yoo, J. 72

Yoon, H.S. 78

Yoshida, H. 78

Youn, S.W. 75, 79

Yu, M. 65

Zlotogorski, A. 71

Zloty, D. 74

Zouboulis, C.C. 57, 69

\section{KARGER}

(C) 2006 S. Karger AG, Basel

Fax +4161306 1234

E-Mail karger@karger.ch

www.karger.com

Accessible online at:

www.karger.com/drm 